\title{
Synchronisation von Digitalisierung, Qualitätssicherung und Assistenzsystemen an Arbeitsplätzen mit geringem Automatisierungsgrad
}

\author{
Tobias Rusch, Amelie Riegel, Michael Hueber, Florian Kerber, \\ Lukas Merkel, Robin Sochor, Laura Merhar, Massimo Romanelli, \\ Zhenqiang Guo, Hermann Klug und Benedikt Stelzle
}

\begin{abstract}
Zusammenfassung
Aufgrund variantenreicher Produktportfolios und steigender Kundenanforderungen im internationalen Wettbewerb stehen KMU vor besonderen Herausforderungen, die durch klassische Automatisierungstechnologien nicht zu lösen sind. Digital vernetzte Assistenzsysteme bieten die Möglichkeit, MitarbeiterInnen bei der Montage individueller Varianten kognitiv und physisch zu unterstützen. Im Projekt
\end{abstract}

T. Rusch $(\square) \cdot$ A. Riegel $\cdot$ M. Hueber $\cdot$ F. Kerber

Technologietransferzentrum Nördlingen, Hochschule Augsburg,

Nördlingen, Deutschland

E-Mail: tobias.rusch@hs-augsburg.de; amelie.riegel@hs-augsburg.de;

michael.hueber@hs-augsburg.de; florian.kerber@hs-augsburg.de

L. Merkel $\cdot$ R. Sochor $\cdot$ L. Merhar

Fraunhofer-Institut für Gießerei-, Composite- und Verarbeitungstechnik IGCV,

Augsburg, Deutschland

E-Mail: lukas.merkel@igcv.fraunhofer.de; robin.sochor@igcv.fraunhofer.de;

laura.merhar@igcv.fraunhofer.de

M. Romanelli · Z. Guo

paragon semvox GmbH, Kirkel-Limbach, Deutschland

E-Mail: romanelli@semvox.de; guo@ semvox.de

H. Klug

SPN Schwaben Präzision Fritz Hopf GmbH, Nördlingen, Deutschland

E-Mail: hermann.klug@spn-drive.de

B. Stelzle

Ohnhäuser GmbH, Wallerstein, Deutschland

E-Mail: benedikt.stelzle@ohnhaeuser.de

(C) Der/die Autor(en) 2021

T. Jeske und F. Lennings (Hrsg.), Produktivitätsmanagement 4.0, ifaa-Edition, https://doi.org/10.1007/978-3-662-61584-3_7 
SynDiQuAss wurden auf Basis einer von den Partnern gemeinsam erarbeiteten und verabschiedeten Handlungsverpflichtung Methodiken und Technologien für den Einsatz an prototypischen Montagearbeitsplätzen entwickelt. Ziel des Projekts ist es, Produktqualität und Arbeitsproduktivität im Bereich der Montage in kleinen und mittelständischen Unternehmen durch Einsatz von Assistenzsystemen und Digitalisierungstechnologien zu verbessern. Während der Konzept- und Entwicklungsphasen wurden Akzeptanzuntersuchungen, Mitarbeiterbefragungen und Validierungsstudien durchgeführt, um die Qualität der Lösungsansätze zu beurteilen und die betroffenen MitarbeiterInnen am Transformationsprozess teilhaben zu lassen. Als finales Projektergebnis wurden zwei spezifizierte Montagearbeitsplätze zur prozessintegrierten robotergestützten Qualitätskontrolle und zur hybriden Getriebemontageassistenz aufgebaut und innerhalb der existierenden Produktionsstätten einem Praxistest unterzogen.

\subsection{Ausgangssituation}

Im Zuge der Digitalisierungsoffensive werden immer mehr Technologien im Bereich der flexiblen Industrieautomation wie etwa frei navigierende fahrerlose Transportsysteme oder kollaborierende Robotersysteme kommerziell verfügbar. Um die daraus resultierenden Potentiale nutzen zu können, müssen neue und einfach anpassbare Einsatzmöglichkeiten in allen Produktionsbereichen geschaffen werden. Trotz der Fortschritte der Innovationsoffensive „Industrie 4.0“ und anderer Initiativen [4, 42] fehlen bislang gerade für KMU Umsetzsetzungsstrategien und methodische Grundlagen dafür.

Insbesondere im Bereich der Montage überwiegen selbst in hochentwickelten Branchen wie dem Automobilbau manuelle Tätigkeiten, die von qualifizierten FacharbeiterInnen durchgeführt werden müssen. Mehrere Faktoren führen dazu, dass die Automatisierung solcher Arbeitsplätze bislang nicht möglich war und auch auf absehbare Zeit technologisch nicht realisierbar erscheint (siehe auch [5]):

Einer der Faktoren ist die hohe Variantenvielfalt des Produktportfolios, die hochflexible und gleichzeitig intuitiv bedienbare Systeme erfordert. Das Problem der kombinatorischen Explosion im Bereich der Massenproduktion und kundenindividuelle Anpassungen in der variantenreichen Serienproduktion mit Losgrößen von 10 bis 1000 Stück erschweren den Einsatz von Standardautomatisierungslösungen wie Industrierobotern, die Vorteile bei der präzisen Ausführung repetitiver Tätigkeiten haben. Zudem ist die Applikationsentwicklung für solche Systeme aufwendig und erfordert qualifiziertes Fachpersonal, um alle Aspekte von den Sicherheitsanforderungen für die 
Gesamtanwendung bis zur Bewegungssteuerung der Einzelkomponenten zu berücksichtigen. Trotz virtueller Simulationstools ist die Inbetriebnahme automatisierter Produktionssysteme oftmals langwierig und arbeitsaufwendig.

Die Komplexität von Montagetätigkeiten resultiert aus der Vielzahl einzelner Arbeitsschritte, der Nutzung unterschiedlicher Werkzeuge und den ergonomischen Anforderungen. So müssen zum Beispiel bei der Getriebemontage Press-, Schraub- und Schlagwerkzeuge mit unterschiedlichen Kraft- und Drehmomentanforderungen verwendet werden sowie Dicht- und Schmiermittel konturabhängig appliziert werden. Das Verlegen von Kabelkanälen oder die Montage schwerer Baugruppen in platzbegrenzten Umgebungen sind weitere Beispiele dafür, dass die Vielfalt von Montagetätigkeiten die Einsatzmöglichkeiten vollautomatischer Produktionslösungen erschwert [43].

An dieser Stelle setzt das Projekt SynDiQuAss an: Am Beispiel realer Anwendungsfälle kleiner und mittelständischer Produktionsunternehmen wird ein standardisiertes Vorgehen entwickelt, um Montagearbeitsplätzte durch digital vernetzte Assistenzsysteme anhand der Bedürfnisse der WerkerInnen so weiterzuentwickeln, dass Produktivität und Produktqualität gleichermaßen gesteigert werden können.

In der Produktionstechnik wird unter dem Begriff „Assistenzsystem“ ein technisches System verstanden, das Informationen über Sensoren und Eingaben aufnimmt, verarbeitet und das Ergebnis in geeigneter Form in den Arbeitsprozess einbringt [22]. Dabei steht gerade nicht die autonome Durchführung von Fertigungsprozessen im Vordergrund, sondern die Unterstützung menschlicher Arbeitskräfte durch gezielte Informationsbereitstellung (kognitive Assistenz) [16], Reduzierung körperlicher Belastungen in Form der motorischen Ausführung von Teilprozessschritten (physische Assistenz) oder eine Kombination in Form einer hybriden Assistenz. In der Literatur sind diverse Untersuchungen $\mathrm{zu}$ Einsatzmöglichkeiten einzelner Assistenzsystemtechnologien beschrieben. Anwendungsszenarien wie die Anlageninbetriebnahme [1] oder lernunterstützende Systeme [10] werden dabei ebenso wie Planungsmethodiken betrachtet, siehe u. a. [3, 17].

Bedürfnisse und Anforderungen der MitarbeiterInnen und des Unternehmens in Einklang zu bringen, also Arbeitsbedingungen, Prozessplanung und Qualitätskontrolle gleichzeitig zu beachten, erfordert eine ganzheitliche Herangehensweise. Das Projekt entwickelt durch eine reflektierte und modellbasierte Synchronisierung von Digitalisierung und Assistenzsystemen Unterstützungsfunktionen, die es Personen mit unterschiedlichen Qualifikationsniveaus sowie unterschiedlichen Fähigkeiten und Fertigkeiten ermöglicht, den neuen und schnell wechselnden Anforderungen gerecht zu werden. Neben den direkten Auswirkungen auf die betroffenen WerkerInnen - geringere Arbeitsbelastung, ergonomische Verbesserungen, höhere Arbeitsmotivation etc. werden auch indirekte sozioökonomische Vorteile erwartet: Dem Facharbeiterrückgang 
durch den demografischen Mangel kann entgegengetreten werden, da Anlernzeiten für ungelerntes Personal reduziert und so flexiblere Einsatzmöglichkeiten z. B. bei Auftragsspitzen möglich werden [2].

Die Qualitätssicherung spielt im produzierenden Gewerbe eine immer wichtigere Rolle. Ein zentrales Ziel dieses Projektes ist es, Qualitätssicherung in die Produktionsprozesse $\mathrm{zu}$ integrieren sowie möglichst weitgehend mit anderen Unternehmensfunktionen sowie Planungs- und Steuerungssystemen zu vernetzen. Es wird ein Stichprobenumfang von $100 \%$ angestrebt, um die Rückverfolgbarkeit bis auf die Ebene des Einzelauftrags bzw. von Einzelstücken zu gewährleisten. Speziell die geringen Losgrößen und die hohe Variantenvielfalt erfordern eine neue, arbeitsplatzbezogene und prozessorientierte Herangehensweise [35], die kundenspezifischen Anforderungen Rechnung trägt [41]. Arbeitsplätze, Qualitätsdaten und Produktionsplanung sollen durchgängig miteinander vernetzt und zur bedarfsgerechten Prozesssteuerung genutzt werden [27].

Die Digitalisierung in der Montage soll zur Synchronisierung der Anforderungen der MitarbeiterInnen und des Unternehmens dienen. Denn ganzheitliche Lösungen für einzelne Montagearbeitsplätze sind bisher weder im Forschungsstadium detailliert untersucht worden, noch haben sie Einzug in die unternehmerische Praxis gefunden. Studien behandeln lediglich Teilaspekte wie den Einsatz von einzelnen physischen oder kognitiven Assistenzsystemen. Die hohe Variantenanzahl und geringe Losgrößen in der Montage der Industriepartner erfordern häufiges Umrüsten der Handarbeitsplätze und erschweren eine Standardisierung der Arbeitsprozesse. Die Herstellung dieser Standardisierung bzw. Systematisierung in der Montage sind wichtige Ziele im Projektverlauf [4]. Der im Projekt gewählte Lösungsansatz liegt in der durchgängigen Prozessmodellierung, für die verschiedene Ansätze untersucht [29, 32] und problemspezifisch eingesetzt werden.

\subsubsection{Vorstellung der Anwendungsfälle}

Bei der Auswahl der Anwendungsfälle wurde in enger Kooperation zwischen wissenschaftlicher Seite und Industriepartnern darauf geachtet, dass die Use-Cases real existierende Produktionsprozesse abbilden und sämtliche Veränderungen bzw. Projektergebnisse in diese Prozesse integriert werden können. Die Arbeitsplätze beider Anwendungsfälle weisen vor Projektbeginn einen geringen Grad an Digitalisierung auf, physische oder kognitive Assistenzsysteme sind an den betrachteten Arbeitsplätzen nicht im Einsatz. Die Losgrößen bewegen sich im ein- bis zweistelligen Bereich, während die Variantenanzahl bei bis zu 1000 liegt. Diese Eigenschaften der Use-Cases spiegeln die oben beschriebenen Herausforderungen wider. 
Für den Bereich der synchronisierten Qualitätssicherung wurde der Montagearbeitsplatz für Videoarme als Komponenten von Flugzeugsitzen beim Partner Ohnhäuser GmbH ausgewählt. Die Ohnhäuser $\mathrm{GmbH}$ ist ein mittelständisches Unternehmen in Wallerstein im Ries. In der über 60-jährigen Firmengeschichte hat sich der Betrieb als Zulieferer von Bauteilen und Baugruppen für die Luftfahrt und andere Industriebereiche etabliert. Die über 300 hoch qualifizierten MitarbeiterInnen verteilen sich auf die fünf Kernbereiche CNC-Fertigungstechnik, Werkzeug- \& Vorrichtungsbau, Blechbearbeitung, Baugruppenherstellung \& Systementwicklung und Additive Manufacturing.

Im Bereich der Systementwicklung \& Baugruppenherstellung werden hauptsächlich Komponenten montiert, die in den Unternehmensbereichen CNC-Fertigungstechnik und Blechbearbeitung hergestellt werden. Einer der Hauptkunden ist die Firma RECARO Aircraft Seating GmbH \& Co. KG mit Sitz in Schwäbisch Hall. Für diesen Kunden fertigt die Ohnhäuser $\mathrm{GmbH}$ unter anderem Videoarme für Flugzeugsitze.

Die verbauten Komponenten für die Videoarme müssen nicht nur funktionalen, sondern auch ästhetischen Ansprüchen genügen. Da sich die Videoarme größtenteils im Sichtbereich des Fluggastes befinden, werden auch erhöhte Ansprüche an die Oberfläche der Komponenten gestellt. Dies fordert von den MonteurInnen nicht nur Sorgfalt im Handling der Bauteile sondern auch erhöhte Aufmerksamkeit und ein geschultes Auge zur Erkennung von kleinsten Abweichungen.

Die Videoarme werden bei Ohnhäuser in einem Arbeitssystem im U-Layout montiert. Sonderkomponenten der Baugruppe werden auftragsbezogen kommissioniert, Standardbauteile sind direkt im Arbeitssystem bevorratet. Eine Herausforderung ist die enorme Variantenvielfalt, von über 1000 verschiedenen Videoarmen die in dem Arbeitssystem gebaut werden (Abb. 7.1).

Die WerkerInnen erhalten ihre Bauunterlagen bestehend aus Zeichnungen und Stücklisten in Papierform und montieren anhand dieser Dokumentation die verschiedenen Varianten. Die Auswahl der passenden Werkzeuge und Montagereihenfolge erfolgt durch die WerkerInnen selbst. Dies erfordert bei der hohen Varianz viel Knowhow.

Eine besondere Anforderung ist das Einstellen der Verstellkräfte der Drehgelenke des Videoarms. Die Kräfte werden durch die MitarbeiterInnen mittels einer Kraftmessdose an festgelegten Messpunkten manuell gemessen und eingestellt. Diese Arbeit erfordert viel Erfahrung und Fingerspitzengefühl, um reproduzierbare Ergebnisse zu erzielen, da die Kraft stark von der Richtung und Geschwindigkeit des Messvorgangs abhängt.

Hauptziel dieses Anwendungsfalls ist die Unterstützung und Entlastung der WerkerInnen durch kognitive als auch physische Assistenzfunktionen. So sollen Informationen wie Zeichnungen und Stücklisten digital verfügbar gemacht werden. Die Erstellung der Arbeitsanweisungen für die MonteurInnnen darf für die Führungskräfte im Bereich Montage keinen zusätzlichen Aufwand verursachen. Durch die Nutzung von vorhandenen Daten aus der Stückliste ist eine automatisierte Erstellung geplant. 


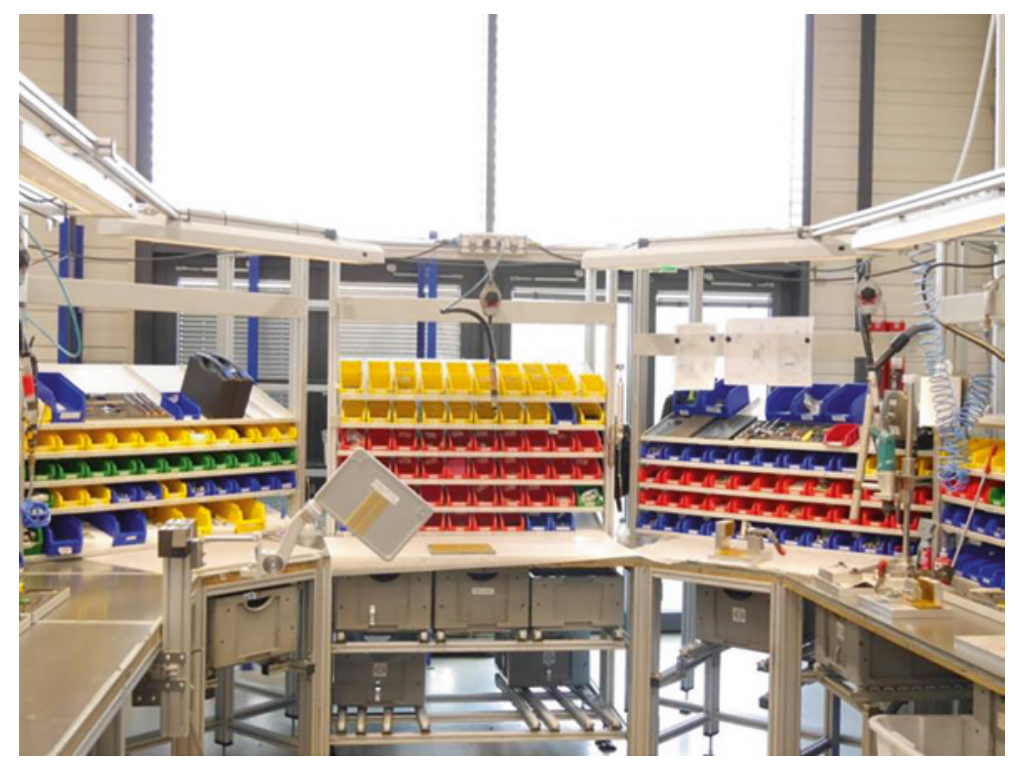

Abb. 7.1 bisheriger Arbeitsplatz bei Ohnhäuser GmbH.

Die Messung der Verstellkräfte kann wiederholgenau durch einen Roboter ausgeführt werden und die zugehörige Auswertung und Dokumentation direkt digital erfolgen. Als Nebenprodukt des Projekts soll unter dem Stichwort Wissensmanagement auch die nachhaltige Sicherung des Knowhows der WerkerInnen in digitaler Form erreicht werden. MitarbeiterInnen müssen nicht nur nach vorgegebenen Arbeitsanweisungen arbeiten, sondern diese durch die Eingabe von Kommentaren verbessern und ergänzen können. Zur Verbesserung der Arbeitsanweisungen und Veranschaulichung von speziellen Tätigkeiten ist es geplant, dass diese durch die WerkerInnen selbst aufgenommenen Bilder ergänzt werden können. Auf diese Weise kann das Wissen der erfahrenen MitarbeiterInnen beispielsweise für das Einlernen neuer MitarbeiterInnen verfügbar gemacht werden. Dadurch, aber auch durch weitere Qualitätssicherungsmaßnahmen soll das Qualitätsniveau nachhaltig gehalten bzw. verbessert werden (Abb. 7.2).

Die im Projekt erarbeitete Neugestaltung des Arbeitsplatzes wird in Abschn. 7.3.3.4 genauer dargestellt. Die Schnittstellen zwischen physischer und kognitiver Assistenz werden durch einen von paragon semvox entwickelten Editor (Abschn. 7.3.4) gewährleistet.

Im zweiten Anwendungsfall bei SPN Schwaben Präzision GmbH wird die Montage einer Baugruppe innerhalb eines Getriebes betrachtet. SPN ist ein mittelständischer Metallverarbeitungsbetrieb in Nördlingen. Ausgehend von der Fertigung von hochpräzisen Uhren in Glashütte/Sachsen umfasst das Produktportfolie noch heute präzise Verzahnungstechnik. Die Firma hat in der Kleinstadt Nördlingen seit 1945 ihren einzigen 
Sitz und beschäftigt inzwischen ca. 300 Mitarbeiter/innen am Standort in der Entwicklung, Fertigung und Montage von Getriebeteilen und kompletten Getriebeantriebssystemen für verschiedene Anwendungen in diversen Industriebranchen. So werden u. a. Getriebeteile und Systeme in Kleinserien für die internationale Textilmaschinenindustrie als auch für den weltweiten Einsatz in der Luftfahrt-Industrie nach höchsten Qualitätsanforderungen hergestellt. Daher ist das Unternehmen auch nach ISO 9100 (Luft- und Raumfahrtnorm) zertifiziert.

Die Produktionsprozesse sind auf Grund der extrem hohen Anzahl an kundenspezifischen Getriebevarianten oft nicht variantenspezifisch optimiert, da die hauseigene Konstruktions- und Entwicklungsabteilung aus Wettbewerbsgründen in immer kürzeren Zeitzyklen für den Kunden optimierte Lösungen plant. Die sich daraus ergebenden geringen Losgrößen bis hin zu Einzelstücken von Sonderanlagen bedingen naturgemäß wenig optimierte Montageprozesse und Qualitätssicherungsmaßnahmen (siehe Abb. 7.3). Für die Produktentwicklung werden bereits digitale Entwurfsverfahren eingesetzt, um durch FEM-Simulationen Bauteilauslegungen zu verbessern. Die Erzeugnisse werden in eigenständig entwickelten Prüfständen verschiedenen Belastungsszenarien unterzogen, um die Berechnungsergebnisse zu validieren und die Produktqualität sicherzustellen. In der Vorfertigung für die Getriebeeinzelteile wie Gehäuse, Ritzelwellen, Zahnräder oder Schneckenräder werden modernste CNC-Bearbeitungsmaschinen eingesetzt, die von im Hause ausgebildeten Facharbeitern bedient werden. Auch hier kommen hochgenaue Messverfahren zur laufenden Qualitätskontrolle zum Einsatz. Die manuellen Montageprozesse dagegen wurden bisher kaum systematisch abgesichert. Die Auswertung von Mängelanzeigen und Kundenreklamationen lieferte keine signifikanten Zahlen von

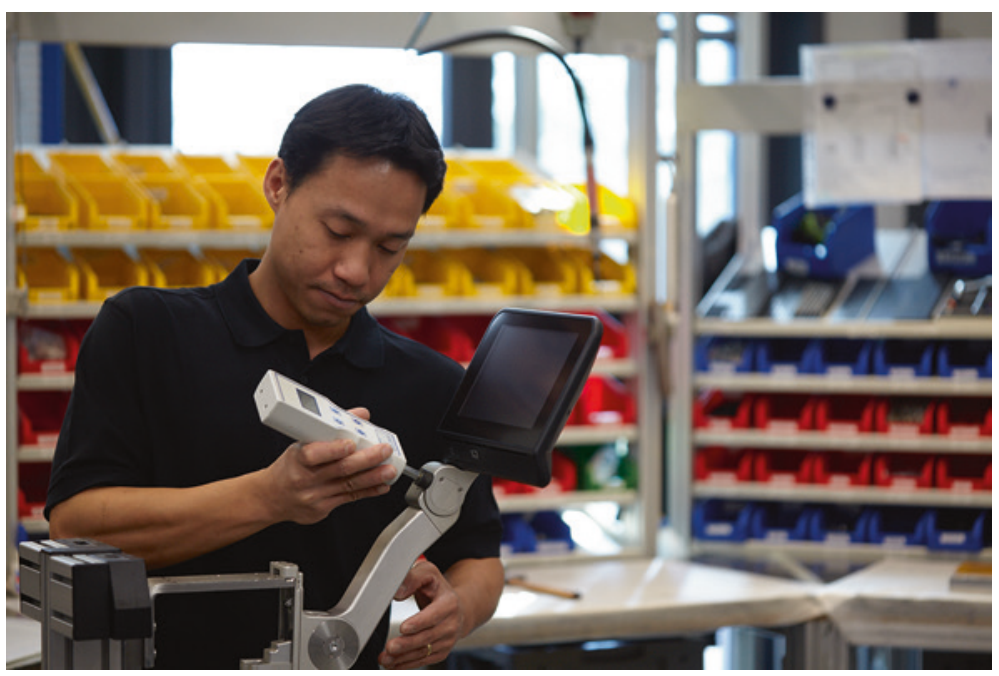

Abb. 7.2 Arbeitsschritt "Kraftmessung" bei Ohnhäuser GmbH 


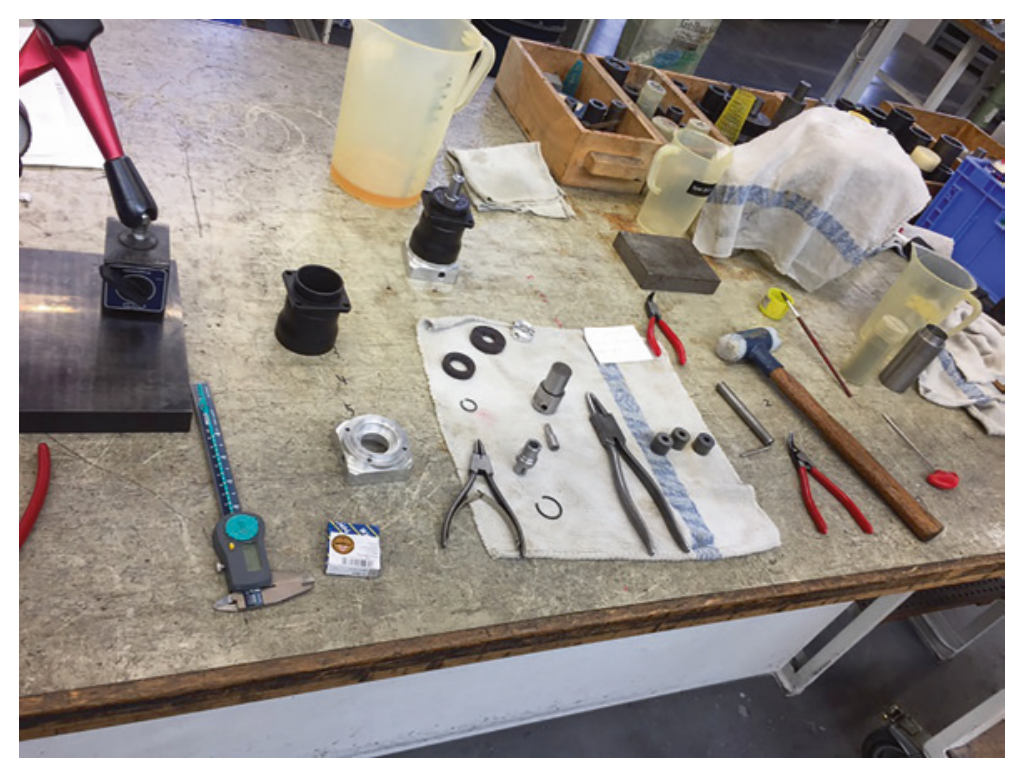

Abb. 7.3 Arbeitsplätze vor der Neugestaltung bei SPN Schwaben Präzision Fritz Hopf GmbH

Qualitätsabweichungen, die der Montage zugeordnet werden konnten, was auf die hervorragende Ausbildung und langjährige Erfahrung des motivierten Fachpersonals zurückgeführt wurde.

Im Rahmen der stetig steigenden Qualitätsanforderungen bei gleichzeitiger Auslegung nahe des physikalischen Grenzbereichs der Belastungsfähigkeit von Bauteilen auf immer engerem Raum sowie massiv gesteigerter Anforderungen an Qualitätsdokumentation und Rückverfolgbarkeit von Mängelursachen durch die Kunden, Auditoren und Zertifizierungsinstitute lassen sich jedoch in der manuellen Montage noch erhebliche Verbesserungspotentiale erzielen. Zudem wird durch den demografischen Wandel und den allgemeinen Fachkräftemangel in der industriellen Fertigung die stetige Verfügbarkeit von erfahrenem und gut ausgebildetem Personal zukünftig schwieriger. Das Montage-Know-how wird von den älteren MitarbeiterInnen oft nicht mehr weitergegeben, da der Zwang zu extrem kurzen Einarbeitungszeiten und hochflexiblen Einsatzbedingungen der MitarbeiterInnen die Einarbeitungszeiten stetig verkürzt. So steigt der Bedarf an operativer Assistenz und der Optimierung der Montagearbeitsplätze in diesem Bereich ständig. Ohne assistierte Produktionsoptimierung durch Digitalisierung und Qualitätssicherungsmaßnahmen an Montagearbeitsplätzen sind Produktivitätssteigerungen und Qualitätsverbesserungen bei internationaler Wettbewerbsfähigkeit der Handarbeitsplätze im Hochlohnland Deutschland nicht möglich.

Bei der Auswahl des Anwendungsbeispiels wurde darauf geachtet, dass der Montageablauf mehrere verschiedene Arbeitsprozesse beinhaltet. Dabei sollten diese Montageprozesse in der gesamten Montageabteilung häufig auftreten und für die Mitarbeiter kognitiv und/oder physisch stark belastend sein. Ein weiteres Kriterium war die zu 
erwartende Zeitersparnis bzw. Produktivitätssteigerung bei der Umsetzung durch den Einsatz der Assistenzsysteme in einzelnen Montageabschnitten. Im Fokus standen hier die Einsatzmöglichkeiten eines kollaborierenden Roboters als physisches Assistenzsystem, da der dafür benötigte Arbeitsraum einen direkten Einfluss auf die Gestaltung des Arbeitsplatzes einnimmt.

Zusammen mit leitenden MitarbeiterInnen aus den Bereichen Montage, Qualitätssicherung, Produktplanung und Arbeitsorganisation wurde hierzu eine Arbeitsgruppe gebildet und eine Aufstellung an verwendbaren Prozessen erstellt. Mit Hilfe eines Fragebogens wurden die vorliegenden Prozesse dann priorisiert und mögliche Produktfamilien ausgewählt. Aus den gewonnenen Ergebnissen wurde das in Abb. 7.4 dargestellte Getriebe mit dem entsprechenden Montageablauf ausgewählt.

Für diesen Anwendungsfall wurde ein Testarbeitsplatz am Technologietransferzentrum Nördlingen aufgebaut (Abschn. 7.3.3.3). Der komplexe Arbeitsvorgang mit vielen kleinteiligen Bauteilen weist teilweise ergonomisch unvorteilhafte Körperstellungen und gefährliche Temperaturen auf. Daher wird wie auch beim Ohnhäuser-Anwendungsfall eine Kombination aus kognitiven und physischen Assistenzsystemen angestrebt. Besonders für die körperlich schweren Arbeiten wird ein Mensch-Roboter-Kollaborierendes System installiert. Über die kognitiven Assistenzsysteme werden einzelne Arbeitsschritte multimodal und interaktiv angezeigt.

\subsection{2 Übergreifende Projektziele}

\section{Handlungsverpflichtung und partizipatives Modell}

Die Akzeptanz der MitarbeiterInnen gegenüber den eingesetzten Assistenzsystemen soll im Projektverlauf eine entscheidende Rolle spielen. Daher wird die Implementierung
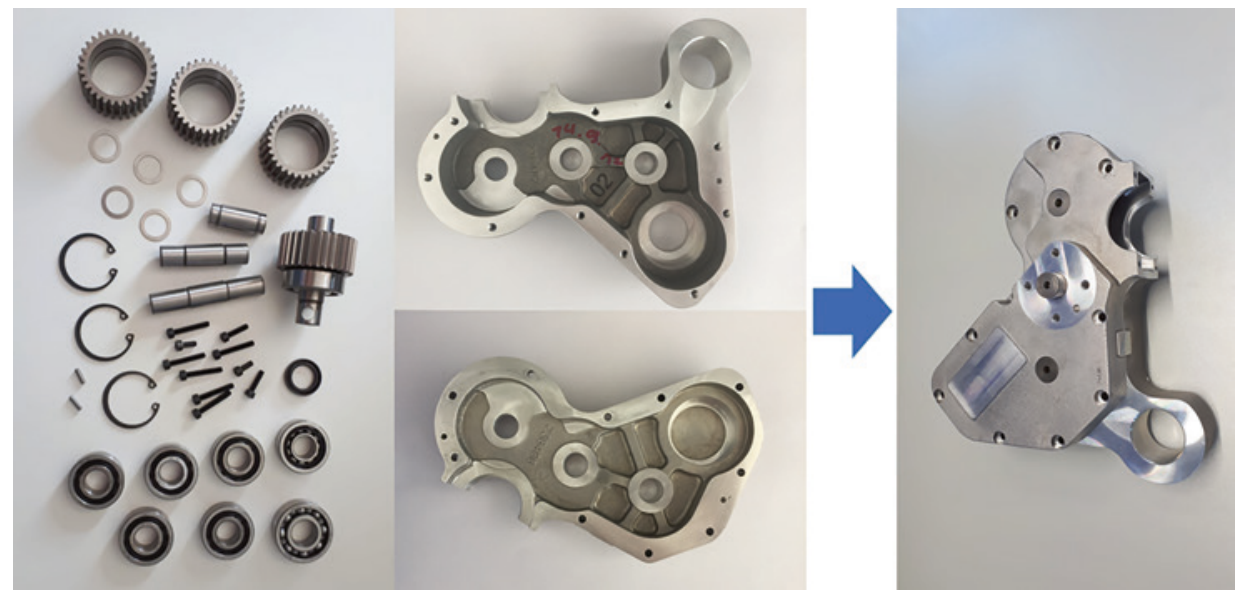

Abb. 7.4 Anwendungsfall bei SPN Schwaben Präzision Fritz Hopf GmbH 
der Assistenzsysteme durch ständige Evaluierungen durch die wissenschaftliche Seite begleitet. Durch dieses partizipative Entwicklungsvorgehen (Abschn. 7.3.1.1) sollen die Bedürfnisse und Vorschläge der MitarbeiterInnen einbezogen werden. Dafür ist eine längere Evaluationsphase vorgesehen, in der die entwickelten Demonstrationssysteme bei den beiden Industriepartnern und den Demonstrations- und Transferzentren der wissenschaftlichen Partner getestet werden sollen. Im Vordergrund stehen hierbei, neben der grundsätzlichen Funktionalität, v. a. die Schnittstellen der Mensch-Maschine-Interaktion. Das partizipatorische Modell soll als Handlungsverpflichtung aller Projektpartner (Commitment) erarbeitet und verabschiedet werden.

\section{Spezifikation von standardisierten Montagearbeitsplätzen}

Durch Standardisierung der Arbeitsplätze und -prozesse lassen sich Zeit- und Kostenvorteile realisieren. Weitere Einflüsse betreffen die Arbeitsplatzgestaltung und die Flexibilität und Wandlungsfähigkeit der Montage(-prozesse). Gerade Flexibilität und Wandlungsfähigkeit in Fertigungsprozessen sind Merkmale einer vernetzten, adaptiven Produktion. D. h. sie passt sich selbstständig an und kann sich gegebenenfalls optimieren. Dies ist bei kleinen und mittelständischen Unternehmen, wie den beiden Industriepartnern im Projekt, kaum oder gar nicht zu finden [21]. Als Methode kommt eine Prozessmodellierung auf Grundlage der UML zum Einsatz, die zudem als Spezifikation für das Softwaresystem zur Steuerung der Assistenzfunktionen an den Demonstratoren dient. Das Konzept dieser aus Editor und Assistenten bestehenden Software wird in Abschn. 7.3.4.1 beschrieben. Für erfahrene WerkerInnen hält die Software einen Expertenmodus bereit, der nur besonders wichtige Arbeitsschritte mit speziellen Arbeitsbedingungen aufzeigt. Das soll zum einen verhindern, dass erfahrene MitarbeiterInnen in ihrem Workflow eingeschränkt werden und zum anderen die Akzeptanz gegenüber dem Assistenten steigern. Demgegenüber steht ein Modus für unerfahrene MitarbeiterInnen zur Verfügung, der jeden einzelnen Arbeitsschritt mit sämtlichen Hilfestellungen anzeigt. Hilfestellungen sind in diesem Fall Videos, Konstruktionszeichnungen und Informationen, die im Sinne des Wissensmanagements kontinuierlich von MitarbeiterInnen der Montage eingepflegt werden. Dadurch sind keine erfahrenen MitarbeiterInnen für die Einlernphase erforderlich und die Qualitätsstandards können eingehalten werden.

\section{Auswahlkriterien und Implementierungsstrategien für Assistenzsystemtechnologien}

In den letzten Jahren hat sich die Zahl kommerzieller Anbieter von Assistenzsystemen für die Produktion drastisch erhöht. Dies umfasst Hersteller von Montagetechnik, die ihre Hardware zusätzlich mit Software ausstatten. Aber auch MES-Anbieter dringen weiter in den Bereich manueller Anwendungsfälle wie der Montage vor. Zusätzlich entstehen neue Startups im Bereich Assistenzsysteme und mobiler Datenerfassung.

Für die Auswahl geeigneter Systeme müssen potentielle Anwender Kriterien zur Verfügung gestellt bekommen, die zu einer systematischen Bewertung und Auswahl geeigneter Systeme führen. Nur so können Akzeptanz und Wirtschaftlichkeit sichergestellt werden. 
Nach der erfolgreichen Auswahl stellt die Integration der gewählten Systeme und Technologien hohe Anforderungen. So bieten kollaborierende Roboter verschiedene Interaktionsszenarien, die jeweils unterschiedliche Absicherungsmaßnahmen und Implementierungen, z. B. in Form von Abstands- und Geschwindigkeitsüberwachungen, erfordern [24].

\section{Produktivitätssteigerungen und Verbesserungen der Produktqualität}

Für beide Industriepartner sind eine Wirtschaftlichkeitsbetrachtung und eine Messung der Produktivität innerhalb des Projekts von großer Bedeutung. Die Projektergebnisse sollen von den Industriepartnern nach Projektende weiterverwertet werden, indem sie dauerhaft in die Produktionssysteme integriert und die langfristigen Zielsetzungen hinsichtlich Arbeitsbedingungen, Produktivität und Qualität realisieren. Für die Anwendungsfälle werden auch Produktivitätsmessungen durchgeführt. Dabei werden Faktoren wie Arbeitszeit, Transparenz, Risiko, Flexibilität, Arbeitsplatz, Ergonomie und Akzeptanz betrachtet. Erhoben werden die Daten durch verschiedene Methoden, wie beispielsweise der Methods-Time-Measurement- Methode zur Bewertung der Arbeitszeit oder eine Risikobewertung durch eine Failure Mode and Effects Analysis (FMEA). Dieses Vorgehen wird in Abschn. 7.2.3 genauer vorgestellt.

Weitere übergreifende Zielsetzungen betreffen die Arbeitsplatzgestaltung und Qualifizierung der Mitarbeiter. Durch die technischen Möglichkeiten kann das System neben Arbeitsanweisungen oder Stücklisten für die Montage auch zur Qualitätssicherung und Dokumentation des Prozesses beitragen. Die Anbindung eines Kamerasystems kann beispielsweise einzelne Montageschritte auf Fehler überprüfen und diese in Form von Videos oder Bildern - zugeordnet zum speziellen Auftrag bzw. Produkt - dokumentieren. Auch Daten, wie Montagezeiten oder häufiger auftretende Mängel, können gespeichert, ausgewertet und für KVP-Vorhaben genutzt werden. Durch Anbindung z. B. an ein ERP-System kann die Transparenz über den Auftragsstatus oder Produkteigenschaften erhöht werden.

Die entwickelten Demonstratoren sollen außerdem die Einarbeitung neuer MitarbeiterInnen unterstützen und damit erfahrene Mitarbeiter in ihrer Anlerntätigkeit entlasten. Indem letztere in den Entwicklungsprozess eingebunden werden und auch später Informationen ins System einpflegen können, wird zusätzlich ein Wissensmanagement geschaffen, das Erfahrungswissen auch von ausscheidenden Mitarbeitern bewahren kann. Zudem erlernen die MitarbeiterInnen Medienkompetenz im Umgang mit neuen Technologien, was weitere Digitalisierungsvorhaben begünstigen kann.

Nicht zuletzt verfolgt die Einbindung eines Assistenzsystems das Ziel, die Arbeitstätigkeit $\mathrm{zu}$ verbessern. Durch weniger Suchen, multimedial aufbereitete Arbeitsanweisungen, schnellen und einfachen Zugriff auf Zusatzinformationen, wie technische Zeichnungen oder Produktdatenblätter, sowie durch Kommunikationsmöglichkeiten mit 
KollegInnen aus anderen Abteilungen wird die Tätigkeit am Arbeitsplatz erleichtert und verbessert.

\subsection{Vorgehen im Projekt}

Grundlage für die Zieldefinition und Zielerreichungsgrade im Projekt SynDiQuAss waren die Anwendungsfälle der Industriepartner. Daran konnten exemplarisch für kleine und mittelständische Unternehmen Aspekte der Arbeitsplanung, Arbeitsplatzgestaltung und Arbeitsorganisation im Bereich der manuellen Montage analysiert sowie Konzepte und Methoden für die Integration von digital vernetzten Assistenzsystemen entwickelt und umgesetzt werden.

\subsubsection{Vorgehensweise zur Zielerreichung für Unternehmen}

Im Sinne des agilen Projektmanagements bzw. modellbasierten Entwickelns wurde innerhalb des Projekts ein iteratives Vorgehen realisiert: Beginnend mit der Aufnahme und Analyse des Ist-Zustands an den realen Arbeitsplätzen der Industriepartner wurden innerhalb einzelner Arbeitsgruppen für die Anwendungsfälle Projektziele definiert. Die Bearbeitung der einzelnen Anwendungsfälle wurde von jeweils einem Vertreter der beteiligten Forschungs- und Industriepartner koordiniert. Die Synchronisation der verschiedenen Untersuchungsgegenstände, d. h. insbesondere die Modellierung von Montageprozessen, die Auswahl und Integration unterschiedlicher Technologien zur Montageassistenz sowie die systematische Definition von Qualitätsprozessen, wurde durch einen regelmäßigen Austausch innerhalb des Forschungsverbunds (Gesamtprojekttreffen, Workshops, Jour-fixes) erzielt.

Die entwickelten Lösungen wurden als Teilmodule in separaten Demonstratoren aufgebaut und getestet. Regelmäßige Abstimmungen zur Kontrolle und Anpassung der Zieldefinitionen und Zielerreichungsgrade sicherten den Rückfluss z. B. aus Befragungen, Workshops und Probandentests während des Projektverlaufs. Als übergreifender Handlungsleitfaden diente das Commitment aller Projektpartner (Abschn. 7.3.1), das als erster Meilenstein realisiert wurde.

Abb. 7.5 veranschaulicht die konkrete Vorgehensweise am Beispiel des Anwendungsfalls der assistierten Qualitätsprüfung beim Industriepartner Ohnhäuser $\mathrm{GmbH}$ (vgl. Abschn. 7.3.3.4). In diesem Fall werden Flugzeugsitzteile in verschiedenen Varianten aus fünf Unterbaugruppen montiert und nach dem Zusammenbau ein Funktionstest der Gelenkkräfte als Qualitätsprüfungsmerkmal durchgeführt. Beginnend mit den Vorortbesichtigungen und der Aufnahme des Ist-Zustands wurde die Dokumentation der Produktfamilie und des Montageprozesses erstellt und während der Laufzeit ergänzt. Hierzu wurden Workshops mit anderen Unternehmensbereichen, z. B. der IT-Abteilung und Qualitätssicherung, durchgeführt, um die Informationen zu sammeln. Aspekte wie 


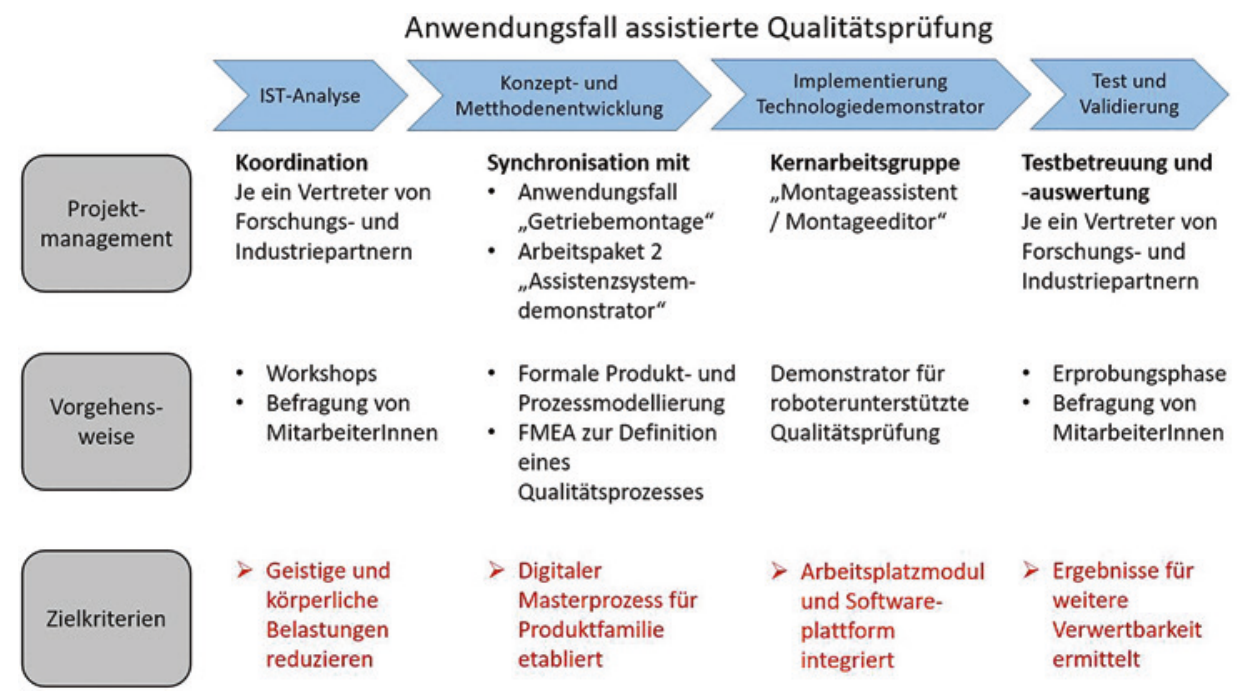

Abb. 7.5 Vorgehensweise am Beispiel eines Anwendungsfalls im Projekt SynDiQuAss

z. B. die körperliche und geistige Belastung der ProduktionsmitarbeiterInnen wurden durch eine Befragungsaktion zum Projektstart ermittelt, um als Bewertungskriterium für den Vorher/Nachher-Vergleich zu dienen. Die unternehmensinterne Kommunikation wurde von der Teilprojektleitung des Partners übernommen. Für die Erstellung einer digitalen Datengrundlage bestehend aus Konstruktionsdaten, Montage- und Prüfanweisungen musste zudem der Kontakt zum Auftraggeber genutzt werden. Auf Basis der Produkt- und Prozessdaten wurde zum einen eine formale Modellierung zur Beschreibung der Produktfamilie entwickelt. Dieser universelle Ansatz soll branchenund produktunabhängig als Masterprozess etabliert werden. Zum anderen wurde auf Basis einer Fehlermöglichkeits- und -einflussanalyse (Prozess-FMEA) ein kompletter Qualitätsprozess definiert, der alle Komponenten, Merkmale und Eigenschaften für die Prüfaufgabe enthält. In dieser detaillierten Prozessanalyse werden für jeden Einzelarbeitsschritt die verwendeten Werkzeuge, Sollzustände der Arbeitsergebnisse als auch Risiken und Folgen von Fehlleistungen definiert. Die Synchronisation mit dem zweiten Anwendungsfall sowie den Ergebnissen aus dem Arbeitspaket 2 (Aufbau eines Assistenzsystemdemonstrators) lieferte ein Konzept für die Realisierung eines hybriden Assistenzsystemarbeitsplatzes. Für die Implementierung des Anwendungsfalls wurde ein spezielles Modul für die roboterunterstützte Qualitätsprüfung aufgebaut. Die Schnittstellen zur zentralen Assistenzfunktionssteuerung, dem von paragon semvox entwickelten Montageeditors, wurden in einer Kernarbeitsgruppe spezifiziert und implementiert. Das Modul wurde schließlich zur abschließenden Erprobungsphase in das Produktionsumfeld des Industriepartners integriert und dort in einem mehrwöchigen Probebetrieb evaluiert. Die Auswertung anhand der vorab definierten 
Bewertungskriterien soll Aufschlüsse für die weitere Verwertung liefern. Zudem soll analysiert werden, welche Entwicklungsschritte für den Einsatz im Produktivbetrieb noch erforderlich sind, z. B. im Hinblick auf die Arbeitssicherheit.

Diese Vorgehensweise lässt sich auch auf andere Anwendungsfälle übertragen. Zentral für die Umsetzung von Innovationen innerhalb eines Unternehmens ist die Beteiligung der MitarbeiterInnen aus allen betroffenen Abteilungen. So lässt sich umfassendes Unternehmens-Know-How bündeln und die Umsetzung beschleunigen. Des Weiteren lässt sich feststellen, dass Motivation und Identifikation der Belegschaft zu Wandel und Erneuerung in der Produktion spürbar gesteigert werden, sobald eigene Ideen und die Expertise der Belegschaft berücksichtigt werden. Aus den Erfahrungen des Forschungsprojektes lässt sich demnach sagen, dass bei Einführung neuer Prozesse oder Änderungen in der Produktion sämtliche an der firmeninternen Wertschöpfungskette beteiligte Abteilungen beteiligt werden sollten. Evaluationen der entwickelten Konzepte und Implementierungen im Probebetrieb sichern das methodische Vorgehen $\mathrm{ab}$, indem Usability von Technologien und Systemakzeptanz in unterschiedlichen Projektphasen ermittelt werden [8].

\subsubsection{Auswahl der Assistenzsysteme mit integriertem Erprobungskonzept}

Das entwickelte Vorgehen zur Auswahl von Assistenzsystemen basiert auf fünf Schritten Abb. 7.6 und ist von anwenden Unternehmen entsprechend der folgenden Beschreibung durchzuführen. Die fünf Schritte bestehen aus einer Vorauswahl, zwei Testphasen mit

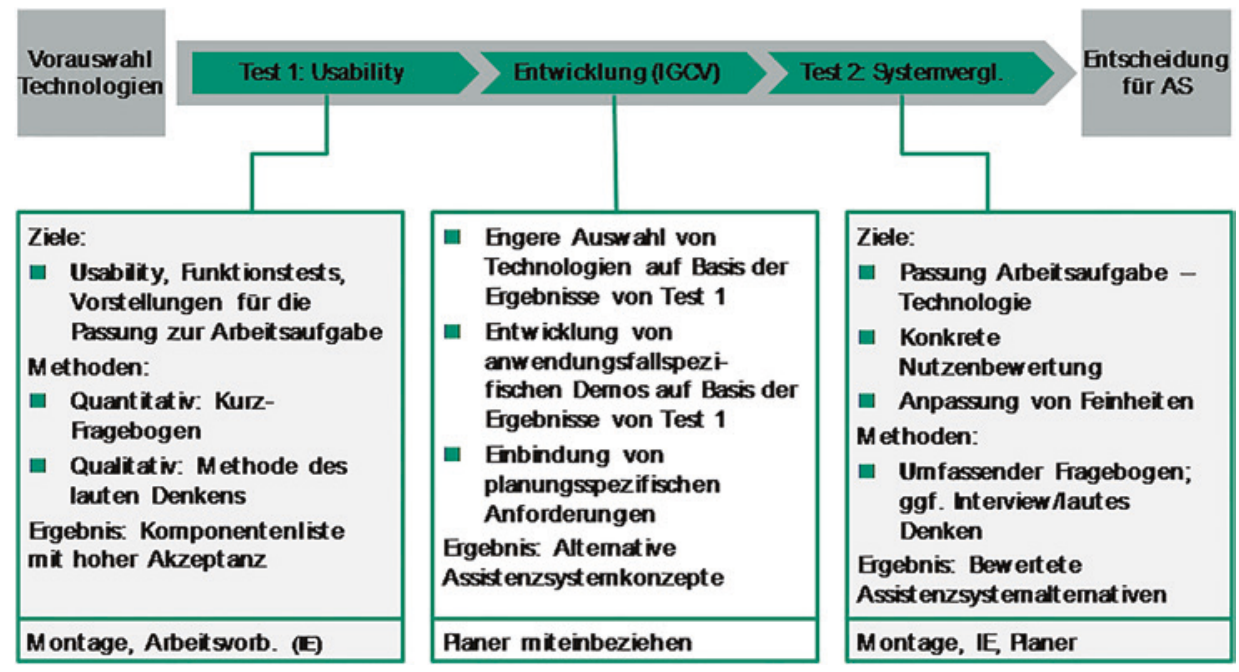

Abb. 7.6 Vorgehen im Projekt zur Auswahl von Assistenzsystemen 
zwischengeschalteter Entwicklungsphase sowie einer finalen Entscheidung für ein Assistenzsystem.

Auf Basis des Anwendungsfalls und Erfahrungswissen wird zu Projektbeginn eine Vorauswahl von Technologien getroffen. Mit diesen Technologien sollen die durch das Assistenzsystem angestrebten Ziele, in den betrachteten Anwendungsfällen Produktivitäts- und Qualitätsverbesserungen, erreicht werden.

Mit der Vorauswahl von Technologien wird die erste Testphase gestartet, die mögliche Hardware-Devices bzgl. ihrer Usability bewerten. Mittels eines Kurzfragebogens und der Methode des lauten Denkens wird Feedback von den zukünftigen Nutzern eingeholt. Ergebnis ist eine Liste an Komponenten für Assistenzsysteme, die eine hohe Akzeptanz aufweisen.

In der darauffolgenden Entwicklungsphase werden auf Basis des ersten Tests, der lediglich Komponenten von Assistenzsystemen umfasst hat, gesamtheitliche Assistenzsysteme konzipiert. Hierbei können auch unterschiedliche Assistenzsysteme entstehen, die alternative Technologien enthalten oder Ausbaustufen voneinander sind.

Im zweiten Test findet ein Vergleich der konzipierten Systeme statt. Mit den zukünftigen Nutzern soll wiederum die Akzeptanz bewertet werden.

In einer finalen Entscheidung werden Management und Controlling eingebunden, um die Nutzen des Assistenzsystems den voraussichtlichen Kosten gegenüberzustellen. Für Kosten und quantifizierbare Nutzen sollen Methoden der Investitionsrechnung (Kapitalwertmethode, Amortisationszeit, etc.) eingesetzt werden. Für nicht quantifizierbare Nutzen (z. B. Steigerung der Attraktivität des Unternehmens, besseres Image durch höhere Qualität) soll eine Nutzwertanalyse eingesetzt werden [7].

\subsubsection{Vorgehen zur Produktivitätsmessung}

Bei der Bewertung der Produktivität der erarbeiteten Arbeitsplätze werden die Faktoren Arbeitszeit, Transparenz, Risiko, Flexibilität, Platz, Ergonomie und Akzeptanz betrachtet.

\subsubsection{Bewertung der Arbeitszeit}

Die Bewertung der Arbeitszeit wird mithilfe der Methods-Time-Measurement-Methode (MTM-Methode) durchgeführt. Die MTM-Methode ist ein Verfahren zur Vorgabezeitermittlung, indem der menschliche Bewegungsablauf bis in kleinste Schritte zerlegt wird. Hierfür wurde der Zeitbedarf für die einzelnen Bewegungsabläufe empirisch ermittelt und in Tabellen erfasst. Die Gesamtzeit einer bestimmten Tätigkeit setzt sich aus der Summe aller relevanten Teilzeiten der notwendigen Grundbewegungen zusammen. Man unterscheidet drei Gruppen von Arbeitsbewegungen: Elementarbewegungen wie Hinlangen, Bewegen, Drehen, Greifen und Loslassen, zusammengesetzte Bewegungen wie Einführen, Lösen, Bein-, Fuß- und Körperbewegungen und Hilfsbewegungen wie Drücken und Augenbewegungen. Die Durchführung der Methode 
erfolgt in zwei Schritten. Zunächst werden die Tätigkeiten in ihre Grundbewegungen zerlegt und alle Einflussfaktoren wie Wege und Genauigkeiten ermittelt. Im zweiten Schritt wird den Grundbewegungen gemäß der MTM Normzeitwertkarte der entsprechende Zeitanteil zugeordnet. Die Zeiteinheit TMU (Time Measurement Unit) entspricht 0,036s. Diese Methode der Arbeitszeitbewertung wurde gewählt, da an den Arbeitsplätzen der Industriepartner zum Teil neue Produkte moniert werden und daher keine Zeiten zum Vorher-Nachher-Vergleich vorliegen [30].

\subsubsection{Risikobewertung}

Zum Projektstart wurden bei den Industriepartnern Failure Mode and Effects Analysis (FMEA) durchgeführt, anhand derer Entscheidungen getroffen wurde, in welchen Bereichen des Montageprozesses Assistenzsysteme zum Einsatz kommen sollen. Zum Abschluss des Projekts wird die FMEA-Analyse erneut durchführen, um festzustellen, ob die umgesetzten Maßnahmen zu einer Risikominderung und damit zu einem erhöhten Qualitätsniveau führen [25]

\subsubsection{Transparenz und Flexibilität}

Wichtige Punkte für die Transparenz eines Prozesses sind die lückenlose Dokumentation des Montageablaufs, aber auch das Änderungs- und Wissensmanagement. Durch die digitalisierten Montageanweisungen mit Rückmeldefunktionen wird die Transparenz erhöht. Allerdings sind mögliche Einbußen in der Flexibilität bei der Einführung neuer Varianten zu untersuchen. Hierfür sind Mitarbeiterbefragungen vorgesehen.

\subsubsection{Platz, Ergonomie und Akzeptanz}

Zur Bewertung möglicher Platzeinsparungen wird der Platzbedarf der ursprünglichen Arbeitsplätze dem der neuen Arbeitsplätze ausgemessen und gegenübergestellt. Zudem soll mit den neuen Arbeitsplätzen auch die Ergonomie verbessert werden. Diese Verbesserung soll wie die Akzeptanz der Mitarbeiter mit der Unified Theory of Acceptance and Use of Technology (UTAUT) Methode durchgeführt. Das UTAUT ist eine allgemeingültige Theorie zur Akzeptanz von Technologien, die aus acht vorhergehenden Modellen entwickelt wurde. Als bestimmende Faktoren werden Leistungserwartung, Aufwandserwartung, sozialer Einfluss sowie unterstützende Bedingungen berücksichtigt. Das jeweilige Geschlecht, Alter, Erfahrung und die Freiwilligkeit der Nutzung nehmen nach dem Modell einen weiteren Einfluss. Mithilfe von Fragebögen ist eine quantitative Bewertung möglich [31].

\subsubsection{Ganzheitliche Wirtschaftlichkeits- und Produktivitätsbetrachtung}

Das strukturierte und planvolle Vorgehen im Forschungsprojekt SynDiQuAss unter der Zielsetzung der Transformation von digitalisierten Assistenzsystemen in den realen Arbeitsprozess der manuellen Montage von Getrieben soll dazu beitragen, die Produktivität der neuen Prozesse zu steigern. Für die Bewertung der Produktivität und 
Tab. 7.1 Methoden zur Produktivitätsmessung

\begin{tabular}{l|l}
\hline Kriterium & Bewertungsmethode \\
\hline Arbeitszeit & MTM-Methode \\
\hline Risikobewertung & FMEA \\
\hline Transparenz und Flexibilität & Mitarbeiterbefragung in der Montage und Arbeitsvorbereitung \\
\hline Platz & Abmessung Arbeitsplatz - Vorher/Nachher-Vergleich \\
\hline Ergonomie und Akzeptanz & UTAUT-Methode \\
\hline
\end{tabular}

Wirtschaftlichkeit der entwickelten Lösungsansätze liefern die in Tab. 7.1 zusammengefassten Kriterien und Bewertungsmethoden quantifizierbare Größen.

Daneben sind jedoch weitere qualitative Kriterien zu berücksichtigen. Ein Beispiel hierfür ist die Effizienz des Know-how-Transfers auf Neueinsteiger unter den Anwendern sowie den Wiedereinstieg in einen Prozess, der über längere Zeit nicht mehr angewendet werden musste. Durch die Software-Assistenz werden klare bildliche, filmische, grafische sowie textliche und sprachliche Informationen unmittelbar auf Wunsch und Anforderung des Anwenders am Montageort zur Verfügung gestellt, ohne dass diese Informationen von verschiedenen Personen und analogen Datenträgern wie Papier, Plänen, archivierten Arbeitsvorgaben beschafft werden müssen. Zusätzliche Informationen wie Montagehinweise werden von den erfahrenen Editoren des digitalen Workflows festgehalten und im Arbeitsprozess den einzelnen MitarbeiterInnen sequenziell auf den betreffenden Arbeitsschritt abgestimmt zur Verfügung gestellt. Die Daten werden im Datennetzwerk gegebenenfalls an mehreren Arbeitsplätzen mit den identischen Produkten parallel dargestellt. So wird eine flexible kurzfristige Erhöhung der Montagekapazitäten bei Bedarf ermöglicht, sofern die logistische Rüstung des Arbeitsplatzes mit den benötigten Bauteilen und Komponenten parallel erfolgt. Bei Ausfall von erfahrenen Einweisern oder anderweitiger Beauftragung dieser Personen kann sich ein noch unerfahrener Mitarbeiter schneller autark mit der Montageaufgabe vertraut machen und erhält alle wesentlichen Informationen für einen standardisierten Fertigungsprozess [36].

Die Handmontage durch den Menschen bedingt grundsätzlich die Möglichkeit von Abweichungen von eingeführten Prozessen, selbst wenn diese Personen sehr gut ausgebildet und erfahren sind. So können Fehlleistungen durch Falschmontage, falsche Reihenfolge oder Unterlassen von Arbeitsschritten bei detaillierter Prozessführung erheblich verringert und die Qualitätssicherung der Prozesse verbessert werden. Die Folgekosten von Fehlleistungen und auch der Imageschaden beim Produktempfänger können nicht immer quantifiziert und in die Gesamtbetrachtung der Produktivität einbezogen werden. Eine erhebliche Reduzierung solcher Risiken und Folgekosten ist durch den Einsatz der digitalen Assistenzsysteme im Rahmen von SynDiQuAss zu beobachten - zumal auch Kunden und andere interessierte Parteien am qualitätsvollen Montageprozess deren Einführung begrüßen, fordern oder in Audits positiv vermerken. 
Die Produktivität der menschlichen Arbeit wird auch maßgeblich gefördert durch ergonomische Bedingungen des strukturierten Systemarbeitsplatzes z. B. durch höhenverstellbare Arbeitstische, Informationsdarstellung in optimaler Arbeitsumgebung, gefahrgesicherte Teilprozesse an Werkstücken mit gefahrbringenden Temperaturen durch kollaborierende Roboter (MRK). Die Produktionsergebnisse der neu gestalteten Arbeitsgänge werden teilweise durch digitale Technologien überwacht. So werden die Ergebnisse der Dichtraupen-Aufbringung unmittelbar nach dem Arbeitsgang auf genaue Positionierung und Unterbrechung der Raupe des Dichtmittels auf der Dichtfläche per Vision-Kamera-Inspektion und nachgeschalteter Bild-Datenauswertungssoftware überprüft. Das Prüfergebnis wird unmittelbar danach auf in Ordnung oder nicht in Ordnung qualifiziert. Dies geschieht im Rahmen von hinterlegten Toleranzgrenzen der Qualitätssicherung. So sind die MitarbeiterInnen nicht nur von der anstrengenden Raupenaufbringung entlastet, sondern auch von der Prüfarbeit und Prüfentscheidung, ob der Vorgang erfolgreich war. Weiterhin wird am Ende des Montageprozesses das aufgeklebte Typenschild mit der eindeutig zugeordneten Serien-Nummer und Artikel-Nummer des gefertigten Produktes per Foto-Auswertung dokumentiert. Somit sind alle vorher erfassten Produkt- und Prozessdaten des Werkstückes einer eindeutigen Serien-Nummer zugeordnet und werden im zentralen Firmen-Datennetzwerk für Rückfragen (Rückverfolgbarkeit) und Fertigungsdokumentation gegenüber dem Kunden oder Auditor gespeichert. Dies erleichtert erheblich die Bearbeitung von Qualitäts-Abweichungsmeldungen (QAM) im Fehlerfall und trägt auch zur wirtschaftlichen Gesamtbetrachtung des Systems bei [20].

Die Auswirkungen der entwickelten Lösungsansätze auf die Produktivität an den umgestalteten Montagearbeitsplätzen können somit erst nach Abschluss der Evaluierungsphase in den Partnerunternehmen unter Berücksichtigung aller qualitativen und quantitativen Kriterien abschließend beurteilt werden.

\subsection{Ergebnisse im Projekt}

Bereits vor Abschluss des Projektes kristallisieren sich vier zentrale Ergebnisse für die systematische Untersuchung und Anwendung von digital vernetzten Assistenzsystemen an Montagearbeitsplätzen heraus. Grundlage für die Projektarbeit ist eine Handlungsverpflichtung aller Partner, die insbesondere eine einheitliche Kommunikationsbasis, ein dialogisches Vorgehen bei der Entwicklung und Implementierung sowie partizipatorische Maßnahmen definiert. Für die geeignete Auswahl und Akzeptanz verfügbarer Technologien wurde eine Bewertungsmethodik für Assistenzsysteme entwickelt und anhand eines Demonstratoraufbaus evaluiert. Die Spezifikation standardisierter Arbeitsplätze für die manuelle Montage sowie die Modellierung der Prozesse liefert die methodische Grundlage für die Applikationsentwicklung synchronisierter Arbeitsplätze. Das Auslegungswerkzeug und die Steuerungsplattform finden sich als Ergebnis in den anwendungsnahen Demonstratoren zur Evaluation in den Industrieunternehmen 
wieder. Weitere Ergebnisse zu Auswirkungen auf die Arbeitsgestaltung ergonomische und gesundheitsförderliche Arbeitssystemgestaltung, Produktivitätswirkung und präzise kalkulierter Wirtschaftlichkeit lassen sich erst nach einer abschließenden Evaluierung benennen.

\subsubsection{Ausarbeitung einer projektübergreifenden Handlungsverpflichtung (Commitment)}

Ein Commitment spiegelt die Bindung und die Zusammenarbeit innerhalb eines Unternehmens, einer Organisation oder eines Konsortiums wider. So wie Unternehmen auf die Bindung der MitarbeiterInnen an ihren Arbeitgeber angewiesen sind, so ist auch das Forschungsprojekt SynDiQuAss auf die Zusammenarbeit der Projektpartner angewiesen. Nur auf diese Weise lassen sich die gesteckten Projektziele erreichen. Daher hat das Konsortium als ersten Meilenstein ein Commitment erarbeitet und verabschiedet, das die Basis für eine verantwortungsvolle Zusammenarbeit innerhalb des Verbunds und der Projektlaufzeit legt. Die Projektpartner verstehen unter diesem Commitment eine Selbstund Handlungsverpflichtung, die eine gemeinsame Verhaltensbereitschaft zum Ausdruck bringt. Sie ermöglicht, dass die Zusammenarbeit im Zuge des Projektes bestmöglich gestaltet wird und die MitarbeiterInnen der Projektpartner gleichberechtigt am Projekt teilhaben können. Bei der Um- und Neugestaltung der Arbeitsplätze werden dadurch die gemeinsam definierten ethischen, sozialen, rechtlichen und wirtschlichen Kriterien respektiert und MitarbeiterInnen der Industriepartner über die Ziele und Werte des Projekts informiert. Das Commitment dient außerdem zur Reflexion der Projektarbeiten [42].

\subsubsection{Dialogisches Vorgehen und Motivation der MitarbeiterInnen}

Unternehmen sind auf die Leistungsfähigkeit und Motivation ihrer MitarbeiterInnen angewiesen. Aus diesem Grund spielt die Mitarbeiterbindung im Zuge des Projekts eine große Rolle. Mitarbeiterbindung kann durch positive Erfahrungen und Erfüllung der Erwartungen an die Arbeit durch das Unternehmen gestärkt werden. Die Maßnahmen der Mitarbeiterbindung können auf einer affektiven, rationalen und normativen Ebene ansetzen und zu positiven Erfahrungen führen. Die affektive Dimension ist im Zuge der emotionalen Verbundenheit der MitarbeiterInnen mit deren gewohnten Arbeitsumfeld zu berücksichtigen. Ein Assistenzsystem erfordert die Zusammenarbeit von Mensch und Maschine und kann folglich nur erfolgreich arbeiten, wenn das System von den MitarbeiterInnen akzeptiert wird.

Radikale Veränderungen der Arbeitsbedingungen können Verunsicherungen und Frustration der MitarbeiterInnen zur Folge haben. Demnach ist es essenziell, dass die Wünsche und Anregungen der betroffenen MitarbeiterInnen während des Projektverlaufs berücksichtigt werden. Die Projektpartner bekennen sich deshalb zu einem dialogischen Verfahren, das die MitarbeiterInnen bei der Einführung von Assistenzsystemen mitein- 
bezieht und sie während und nach Ablauf des Projekts SynDiQuAss über die in diesem Rahmen gewonnenen Erkenntnisse fortlaufend zu informiert. Erste Maßnahme dazu war die Veröffentlichung und Diskussion des Commitments in den beteiligten Unternehmen und Einrichtungen.

Neben einer adäquaten Bezahlung stellen qualifizierte MitarbeiterInnen auch Anspruch auf eine gewisse sinnstiftende Arbeit und Arbeitsumgebung. Diese moralisch-ethische Sichtweise ist in der Literatur auch als normative Dimension bekannt. Sinnstiftende Arbeiten für die MitarbeiterInnen können außerdem einen positiven Einfluss auf den betriebswirtschaftlichen Erfolg für das Unternehmen darstellen. Daher wird im Projekt SynDiQuAss darauf geachtet, die Wertschätzung der Belegschaft der teilnehmenden Industriepartner in den Fokus zu rücken. Gleichermaßen wird von allen beteiligten MitarbeiterInnen großes Engagement erwartet. Sie werden in sämtliche Projektarbeiten miteinbezogen und helfen somit, die gesteckten Projektziele zu erreichen. Nur durch die enge Einbindung der MitarbeiterInnen in das Forschungsprojekt ist es möglich, die jeweiligen realitätsnahen Anforderungen in den Montageabteilungen $\mathrm{zu}$ erfüllen. Ergebnisse aus dieser engen Zusammenarbeit dienen der Sicherung der Arbeitsplätze und der Verbesserung der Arbeitsbedingungen für den/die MitarbeiterIn [34].

Die rationale Dimension, auch kalkulatorische Ebene genannt, spielt ebenfalls eine gewichtige Rolle. Hierbei werden Vor- und Nachteile der verschiedenen Projektphasen miteinander abgewogen. Dabei wird den MitarbeiterInnen der Industriepartnern klar vermittelt, dass eine couragierte Mitarbeit im Projekt eine klare Verbesserung der Arbeitsbedingungen nach sich zieht. Couragierte Mitarbeit heißt in diesem Fall u. a. individuelle Anforderungen und Bedürfnisse von MitarbeiterInnen mitzuteilen, sodass diese, wenn möglich, in die Projektziele integriert werden können [17]. Exemplarisch verdeutlicht dies eine in der ersten Projektphase durchgeführte Befragung zu Belastungen der MitarbeiterInnen in der Montage. Dabei wurden körperliche Belastung z. B. durch ungünstige Körperhaltung, langes Stehen und Halten sowie Heben und Bewegen schwerer Lasten, geistige Belastung durch hohen Konzentrationsbedarf, genaues Detailsehen und hohen Leistungsdruck, Zeit- und Termindruck sowie das Suchen von Teilen und Informationen z. B. bei Qualitätsproblemen erfasst. Verbesserung für alle ermittelten Belastungsarten sind Zielsetzung des Projekts und werden nach Abschluss der Testphase zu Projektende erneut evaluiert.

\subsubsection{Im Commitment definierte Schutzmaßnahmen}

Im Forschungsprojekt sind Partner beteiligt, für die auch die Wirtschaftlichkeit im Fokus steht. Daher liegt es am Konsortium, ausschließlich ausgewählte Arbeitsschritte mit bestimmten Assistenzsystemen zu begleiten und auszustatten. Berücksichtigt werden auf der einen Seite menschliche Stärken wie Intuition, Erfahrung, Flexibilität, subjektives Entscheiden und Urteilen und auf der anderen Seite die Vorteile von Assistenzsystemen. Dazu zählen Wiederholungsgenauigkeit und Präzision, aber auch Präzision bei hohen Geschwindigkeiten und Funktionalität im Dauerbetrieb [9]. Assistenz- 
systeme werden deshalb nicht mit der Zielsetzung appliziert, den gesamten Prozess, sondern ausschließlich eine Teilmenge der Arbeitsschritte zu übernehmen und zu unterstützen. Die Entscheidungsfreiheit liegt dabei ausschließlich bei den MitarbeiterInnen. Die Sicherheit an den Arbeitsplätzen in den Montageabteilungen der Industriepartner muss auch bei Testsystemen und Erprobungen jederzeit gewährleistet werden. Den MitarbeiterInnen werden auch während und nach der Projektlaufzeit höchste Sicherheitsstandards zugesichert. Die in SynDiQuAss eingesetzten Technologien und Methoden dienen dazu, die Gefahren in der Arbeitsumgebung der WerkerInnen zu reduzieren. Dies gilt besonders für den Einsatz von Mensch-Roboter-Kollaborationen. Daher findet die Systematik nach DIN ISO/TS 15.066 Roboter und Robotikgeräte - Kollaborierende Roboter große Beachtung. In dieser Erklärung sind Sicherheitsanforderungen an kollaborierenden Industrie-robotersystemen und die Arbeitsumgebung festgelegt. Sie ergänzt die in ISO 10218-1 und ISO 10218-2 angeführten Anforderungen und Anleitungen zum Betrieb von kollaborierenden Industrierobotern. Es müssen bestimmte Gefährdungen mithilfe einer Risikobeurteilung berücksichtigt werden. Dazu zählen roboterbezogene Gefährdungen wie beispielsweise Robotereigenschaften (Geschwindigkeit, Kraft oder Impuls) und die Position der Bedienperson. Gefahren lauern auch beispielsweise durch Werkstücke, die im unmittelbaren Einzugsgebiet des Roboters liegen. Auch prozessspezifische Gefährdungen wie hohe/niedrige Temperaturen oder Einschränkungen aufgrund der geforderten Verwendung von persönlicher Schutzausrichtung können auftauchen $[2,12]$.

In einem Forschungsprojekt, in dem so eng mit MitarbeiterInnen der Industriepartner zusammengearbeitet wird, lässt sich eine Generierung von differenzierten Informationen über die MitarbeiterInnen nicht verhindern. Die Projektpartner erklären mit diesem Commitment, dass sämtliche personenbezogenen Daten sorgfältig und vertraulich behandelt werden und das Konsortium nur nach schriftlicher Bestätigung des betroffenen Unternehmens verlassen.

\subsubsection{Ergebnisse der ersten Erprobungsphase}

Im Rahmen des Forschungsprojekts SynDiQuAss ist ein Demonstrator für kognitive Assistenzsysteme entstanden, der die Arbeit der Zukunft mit Assistenzsystemen erlebbar macht. Als Beispielprodukte kommen hierbei reale Getriebe des Projektpartners SPN zum Einsatz. In mehreren Workshops wurde ein gemeinsamer Use Case entwickelt. Dieser umfasst die Montage des Planetengetriebes EZ24, das in insgesamt 18 Prozessschritten montiert und geprüft wird. Abb. 7.7 zeigt den digitalen Montagetisch bei einer Erprobung. Die Erprobungsergebnisse können auch auf andere Getriebevarianten übertragen werden.

Der entwickelte Demonstrator beinhaltet marktreife optische, akustische und haptische Ein- und Ausgabesysteme wie beispielsweise Touchscreen, Projektor, Headset, 


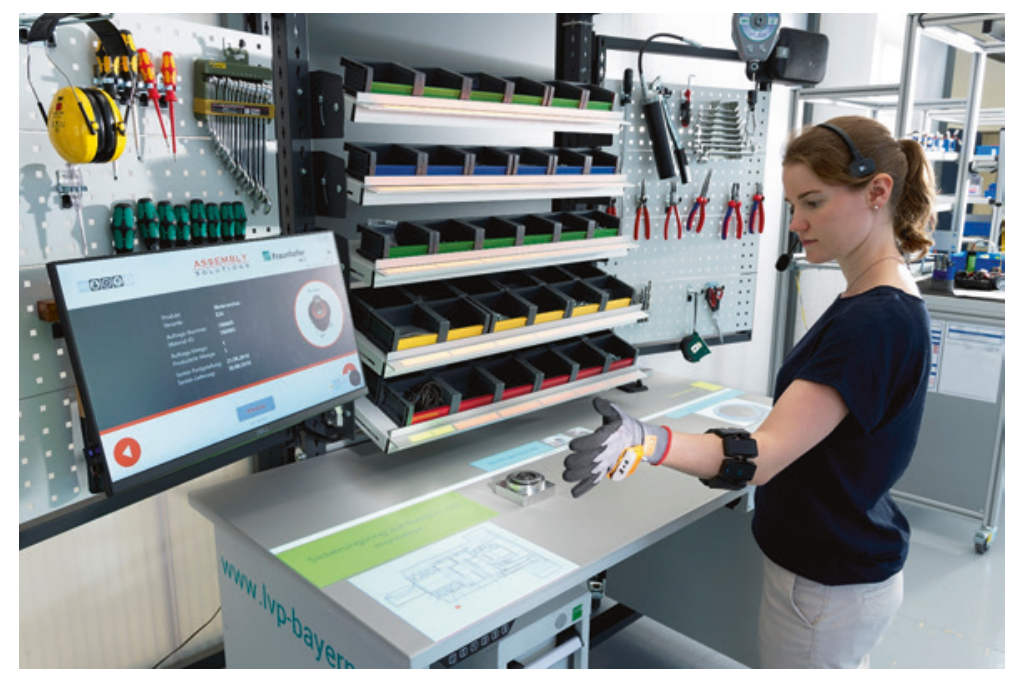

Abb. 7.7 Montagemitarbeiterin bei der Erprobung unterschiedlicher Assistenzsysteme in der manuellen Montage

Proglove und Myo-Gestenarmband. Zudem ist der digitale Montagetisch mobil und kann in jeder Fabrik zu Erprobungszwecken genutzt werden [26].

Das übergeordnete Ziel des Demonstrators ist, durch die Erprobung der Assistenzsysteme im Rahmen einer realen Montage das Unterstützungspotenzial der jeweiligen Systeme erlebbar zu machen und hierdurch die Akzeptanz der Assistenzsysteme nachhaltig zu steigern.

Das Projekt SynDiQuAss bezieht neben technischen Anforderungen auch die Betrachtung des Menschen mit dessen Bedürfnissen mit ein. Besonders bei der Entwicklung soziotechnischer Systeme spielt die Orientierung an Mitarbeiteranforderungen eine bedeutende Rolle [6, 11, 38]. Die Passung des Systems zur Arbeitsaufgabe und die Akzeptanz der Belegschaft können entscheidende Faktoren dafür sein, ob die Einbindung eines Assistenzsystems Erfolg hat oder abgelehnt wird [33, 37]. Im Projekt wird daher ein partizipatives Vorgehen Abb. 7.6, um ein geeignetes und akzeptiertes Assistenzsystem zu entwickeln. Erwartete Vorteile eines partizipativen Entwicklungsvorgehens sind neben der Akzeptanz auch die damit verbundene Bereitschaft, Verantwortung für die Nutzung des neuen Arbeitssystems zu übernehmen, sowie die Nutzung des Erfahrungswissens vor allem langjähriger MitarbeiterInnen für eine optimale Passung zur Arbeitsaufgabe. Mittels des mobilen Demonstrator-Arbeitsplatzes Abb. 7.7 wurden in Schritt zwei bei beiden beteiligten Industriepartnern vor Ort Befragungen der MitarbeiterInnen zur Usability und Akzeptanz einzelner Assistenzsystem-Komponenten durchgeführt. Ziel war es, MitarbeiterInnen über verschiedene Komponenten der Informationseingabe und -ausgabe zu informieren, die Komponenten 
testen zu lassen, und auszuwählen, welche gerne zum Erhalt von Informationen (z. B. Anleitungen) genutzt werden würden. Durch die Kombination eines quantitativen Fragebogens nach dem Test Abb. 7.8 mit der qualitativen Methode des lauten Denkens während des Tests konnten sowohl gezielt Meinungen abgefragt, als auch offene Antworten darüber hinaus generiert werden. Die Variablen Einfachheit der Nutzung, Zuverlässigkeit und Nutzungsintention wurden pro Komponente im Fragebogen mittels Likert Skala von 1 (stimme gar nicht zu) bis 5 (stimme voll zu) abgefragt und anschließend jeweils Mittelwert und Standardabweichung berechnet. Die Ergebnisse wurden für jedes der beiden Unternehmen separat ausgewertet. Trotz eines geringen Stichprobenumfangs von 9-10 Probanden je Unternehmen geben die Ergebnisse Hinweise auf präferierte und abgelehnte Komponenten sowie auf Komponenten, bei denen die Meinungen stark auseinandergehen. Beispielsweise erzielte in einem der beiden Unternehmen das angebrachte Display zur Informationsausgabe bei Abfrage der

\section{Kategorie 1 | Informationsausgabe: Hinweisen und Anleiten}

In den folgenden Fragen geht es um die Bereitstellung von Informationen über unterschiedliche Geräte. Das: sind zum Beispiel Anleitungen auf einem Touch-Display oder Informationen, wie viele Teile aus welchem Behälter für einen Auftrag entnommen werden sollen (Pick-by-light).

Bitte beantworten Sie zu jeder Komponente die drei Fragen unter dem Bild.

\begin{tabular}{|c|c|c|c|c|c|}
\hline \multicolumn{6}{|l|}{ 1. Display } \\
\hline \multirow{2}{*}{ 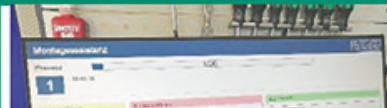 } & \multirow{2}{*}{\multicolumn{5}{|c|}{ Informationen über ein Display erhalten }} \\
\hline & & & & & \\
\hline 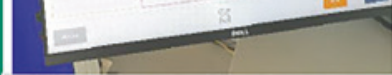 & $\begin{array}{l}\text { stimme gar } \\
\text { nicht zu }\end{array}$ & $\begin{array}{l}\text { stimme eher } \\
\text { nicht zu }\end{array}$ & teils/teils & $\begin{array}{l}\text { stimme } \\
\text { eher zu }\end{array}$ & $\begin{array}{l}\text { stimme } \\
\text { voll zu }\end{array}$ \\
\hline $\begin{array}{l}\text { Ich finde das Display einfach zu } \\
\text { benutzen. }\end{array}$ & $\square$ & $\square$ & $\square$ & $\square$ & $\square$ \\
\hline Das Display funktioniert zuverlässig. & $\square$ & $\square$ & $\square$ & $\square$ & $\square$ \\
\hline $\begin{array}{l}\text { Ich möchte das System gerne zum } \\
\text { Erhalten von Informationen ( } z . B \text {. } \\
\text { Anleitungen) nutzen. }\end{array}$ & $\square$ & $\square$ & $\square$ & $\square$ & $\square$ \\
\hline Anmerkungen: & & & & & \\
\hline
\end{tabular}

Abb. 7.8 Auszug aus dem Fragebogen zur Komponentenbewertung 
Nutzungsintention einen hohen Mittelwert $(\mathrm{M}=4,6)$ bei geringer Standardabweichung $(\mathrm{SD}=0,49)$, während die Informationsausgabe über einen Lautsprecher bei mittlerem Mittelwert $(M=3)$ eine vergleichsweise hohe Standardabweichung $(\mathrm{SD}=1,48)$ erfährt. Die Antworten auf offenen Fragen im Fragebogen sowie die Ergebnisse des lauten Denkens lieferten Hintergründe und weitergehende Anforderungen an das Assistenzsystem. Beispielsweise wurde ein Display nur für Tätigkeiten ohne Öl gewünscht, oder Sprachsteuerung bevorzugt, wenn häufig beide Hände in Benutzung sind.

Auf Basis der Befragungsergebnisse sowie der Ergebnisse vorangegangener Workshops zur Anforderungsanalyse wird in Schritt drei ein Assistenzsystem entwickelt. Im Anschluss daran ist eine weitere Befragung geplant, die das Assistenzsystem nicht in Komponenten aufgeteilt, sondern als ganzes System und in direktem Bezug zur realen Arbeitsaufgabe bewerten lässt. Angelehnt an die Unified Theory of Acceptance and Use of Technology (UTAUT) von Venkatesh, Morris, Davis und Davis (2003) [39] werden dann weitere Faktoren, wie die Erwartung an die Leistung des Systems, die Aufwandserwartung oder der soziale Einfluss, integriert. Wird das System eher negativ bewertet, kann eine qualitative Befragung, beispielsweise mittels Interviews, Hinweise auf nötige Systemanpassungen geben.

\subsubsection{Spezifikation standardisierter Montagearbeitsplätze}

Die Entwicklung neuer Konzepte und alternativer Strategien für eine flexible, mitarbeiterorientierte Fertigung ist notwendig, um Konkurrenzfähigkeit von KMU auf dem Weltmarkt sicherzustellen und Arbeitsplätze im produzierenden Gewerbe zu sichern. Die Montage hat dabei einen ganz besonderen Stellenwert für den Markterfolg und die Produktivität eines Unternehmens. In dieser abschließenden Stufe des Produktentstehungsprozesses (PEP) wird technologisch die Gesamtfunktion des Produktes realisiert [15]. Der PEP besteht aus der eigentlichen Produktentwicklung und der Produktionsentwicklung. Aus der Sicht der IT-Lösungen gehören zum PEP u. a. die virtuelle Produktentwicklung (VPE), die digitale Planung, die Fertigungs- und Montagesimulation sowie das gemeinsame und umfassende Management aller auf das Produkt und die Produktionsplanung bezogenen Informationen in digitaler Form und deren Visualisierung. Als Resultat entsteht ein virtuelles Produkt mit allen zur Herstellung benötigten Planungsunterlagen, die insbesondere die Produktbeschreibung, Spezifikation und das digitale Modell umfassen [14].

\subsubsection{Prozessmodellierung}

Auf dieser Datengrundlage haben sich verschiedene Modellierungsansätze für Montageprozesse entwickelt. Der Montagevorranggraph nach Prenting und Battaglin [29] hat sich dabei für praktische Anwendungen seit langem bewährt. Vorranggraphen beschreiben die Vorgänger-Nachfolger-Beziehungen zwischen den einzelnen Montageschritten und definieren so eine Ordnungsrelation auf der Menge der Montageschritte. Der 
Gozintograph als weitere Darstellungsform ermöglicht zudem eine Verknüpfung der dargestellten Montageschritte mit den Mengenangaben der Stückliste [40].

Einen alternativen Ansatz bietet die Modellierungssprache Unified Modeling Language (UML) [28]. Diese stellt sowohl Strukturmodelle wie das Klassendiagramm als auch Verhaltensbeschreibungen wie das Aktivitätsdiagramm zur Verfügung. Die UML wird dabei mittlerweile nicht mehr als syntaktisch und semantisch vollständig definierte Sprache verstanden, sondern als Sprachrahmen. Erweiterungsmechanismen und semantische Variationsmöglichkeiten erlauben die Definition von Sprachprofilen, die dem jeweiligen Einsatzzweck angepasst werden können [32]. Im Bereich der Industrieautomatisierung hat sich AutomationML als ein solches Erweiterungsprofil etabliert. AutomationML wurde vorrangig für die Nutzung im Bereich des Entwurfs von Produktionssystemen entwickelt und basiert auf XML als Datenformat [13]. XML-basierte Dateiformate erfüllen alle Voraussetzungen für den benötigten Datenaustausch zwischen verschiedenen Entwurfswerkzeugen. Lüder und Schmidt [23] beschreiben die generelle Architektur von AutomationML und die Entwurfsprozesse bezogen auf die Anforderungen der Industrie 4.0.

Auf Basis von UML wird beispielhaft ein Montageprozess unter Zuhilfenahme der Software „Enterprise Architekt“ modelliert. Als Datenbasis fungieren dabei die vorliegenden Produktionsunterlagen in Form der Stückliste, der Zusammenstellungszeichnung und einer rudimentären Montageanweisung. Dabei wird der hierarchische Aufbau der Produktentstehung mit den zur Verfügung stehenden Hilfsmitteln und den notwenigen Fertigungsschritten kombiniert, siehe Abb. 7.9.

Der zeitliche Ablauf des Montageprozesses wird durch ein Verhaltensdiagramm beschrieben. Im Aktivitätsdiagramm der UML werden die einzelnen Montageschritte als Aktivitäten modelliert, deren Zerlegung in Aktionen eine komplette Beschreibung unter Berücksichtigung der Reihenfolge der Teilprozessschritte ermöglicht [18]. Dieses Vorgehen wird im Anwendungsfall auf die Fertigung von Planetengetriebevarianten angewendet. Dabei sind fügende Montageschritte (z. B. Aufsetzen, Einspreizen, ...) sowie Hilfstätigkeiten wie das Fetten von Getrieben dargestellt. Die Teilschritte zur Montage einer Getriebestufe werden variantenabhängig ein- oder mehrmals durchlaufen, was durch die Verzweigungsstelle realisiert ist. Jede Aktivität und somit der gesamte Montageprozess werden wiederum mit elementaren Aktionen beschrieben und mit den zur Durchführung benötigten Ressourcen verknüpft. So stellt beispielsweise die Greifzange die notwendige Ressource für das Einspreizen eines Sicherungsrings dar.

Die Modellierung einer Produktfamilie mittels Klassendiagrammen und die Verknüpfung der Montagschritte zu einem Aktivitätsdiagramm liefert eine Beschreibungsform, die als Grundlage für die Integration von flexiblen Assistenzsystemen zur WerkerInnenunterstützung dient. Die digitale Darstellungsform der UML Diagramme ermöglicht es gerade auch mittelständischen Unternehmen mit einer hohen Variantenvielfalt, moderne Technologien, insbesondere Assistenzsystemen ohne zu großen Anpassungsaufwand an Montagearbeitsplätzen zu integrieren. Um die beschriebenen 


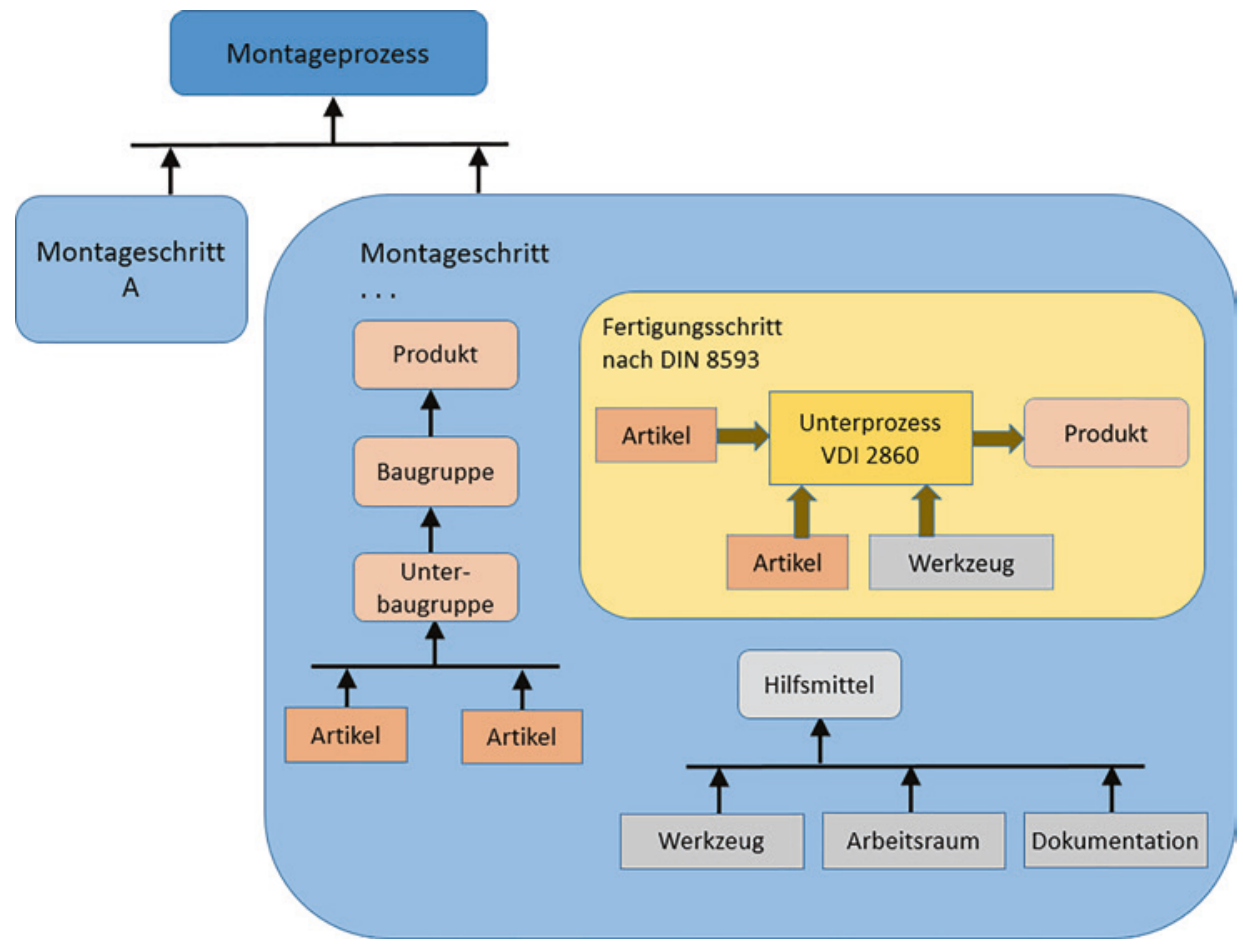

Abb. 7.9 Hierarchischer Aufbau eines Montageprozesses

Modellierungstechniken effektiv anwenden zu können, müssen relevante Konstruktionsdokumente in digitaler Form verfügbar sein. Um den Aufwand zu reduzieren, die das Einpflegen variantenindividueller Arbeitsanweisungen bedeuten würden, wurde ein Plug-in entwickelt, das auf der Grundlage des Klassen- und Aktivitätsdiagramms ein Template für eine Produktfamilie generiert. Innerhalb der Produktfamilien können Anpassungen der Arbeitsanweisungen einfach ausgeführt werden, indem z. B. die verbauten Einzelteile variieren. Das Plug-in verknüpft das Template mit der jeweiligen Stückliste des zu montierenden Produkts und filtert mithilfe einer hinterlegten Logik die für diese Variante benötigten Bauteile. Im Nachhinein kann die Anleitung manuell geprüft werden. Auf diese Weise muss jeweils nur bei der Umstellung auf eine neue Produktfamilie, nicht aber bei der Einführung einer neuen Variante eine neue digitale Anleitung erstellt werden [19].

\subsubsection{Modulkonzept für Montagearbeitsplätze}

Für den mechanischen Aufbau des standardisierten Systemarbeitsplatzes wird ein modularer Aufbau vorgeschlagen. Jedes Modul wird für einzelne Aktivitäten im Montageprozess genutzt. Die einzelnen Module haben ein betriebsspezifisch anpassbares Standardformat und können so leicht ausgetauscht bzw. in ihrer Reihenfolge zueinander 
verändert werden. So kann der Arbeitsplatz bei Bedarf schnell an die wechselnden Anforderungen angepasst und im Rahmen von teilautomatisierten und digital unterstützen Montagekonzepten vernetzt werden. Abb. 7.10 stellt beispielhaft die modulare Aufteilung eines standardisierten Montagearbeitsplatzes dar.

Dieser modulare Aufbau vereinfacht den Einsatz von digitalen Assistenzsystemen stark, da diese nun auf die reduzierten Anforderungen an einem Arbeitsplatzmodul spezialisiert werden können.

\subsubsection{Umsetzung am Beispiel des ersten Anwendungsfalls bei SPN}

Um den Arbeitsbedingungen und dem Arbeitsumfeld in der Montageabteilung für den Anwendungsfall des Partners SPN möglichst nahe zu kommen, wurde bei der Gestaltung des Testarbeitsplatzes versucht, prinzipiell auf die dort vorhandenen Konzepte aufzubauen. Dabei wurde ähnlich den dortigen Systemarbeitsplätzen für die Konstruktion des Arbeitsplatzes ein Rohrverbindungssystem verwendet. Der L-förmige Aufbau ist dabei in drei Arbeitsbereiche unterteilbar.

- Arbeitsbereich 1 besteht aus dem linken Schenkel des Arbeitstisches und besteht aus einer Arbeitsfläche und einem Materialregal. In diesem Arbeitsbereich werden Normund Spezialteilen kollaborativ von MitarbeiterInnen und einem Roboter montiert.

- Arbeitsbereich 2 befindet sich in der Ecke des Aufbaus und dient aus Ausgangsbasis für den kollaborierenden Roboter. Dieser ist auf einem speziellen Roboterwagen montiert, in dessen Sockel die Steuerung des Roboters untergebracht ist. Der Roboter-

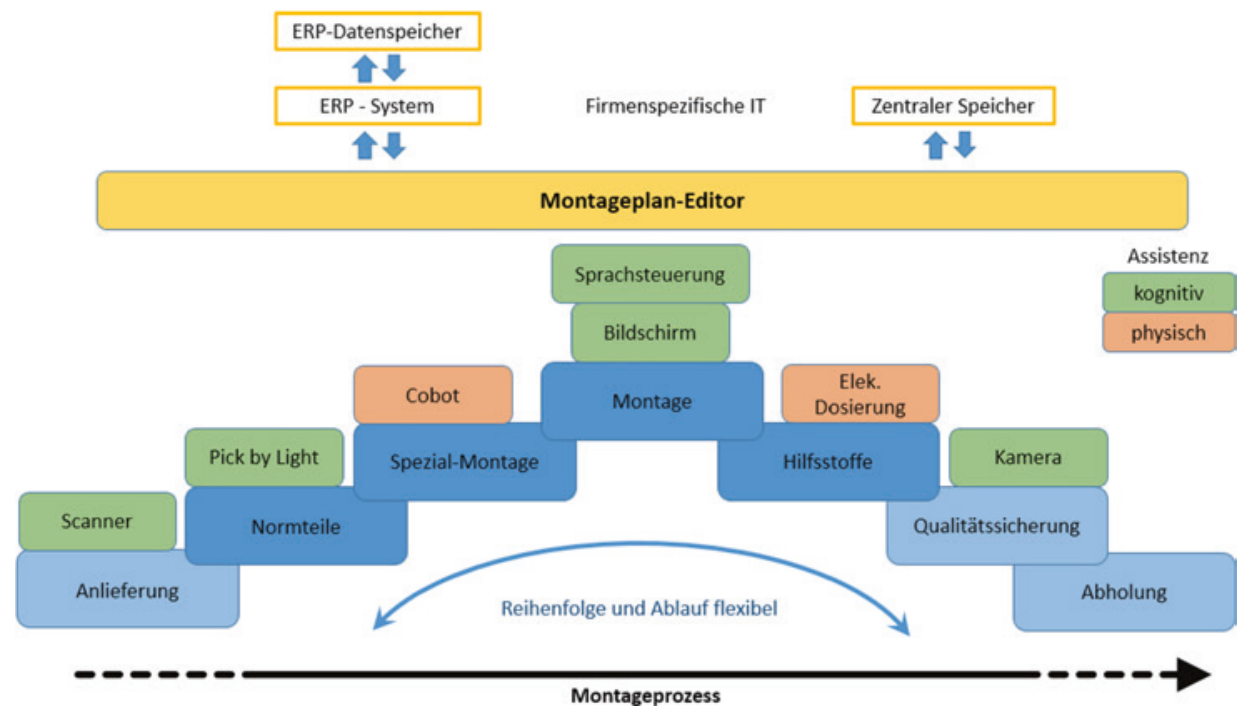

Abb. 7.10 Modularer Aufbau des Montageprozesses (Bild: Tobias Rusch) 
wagen ist fahrbar und kann in Grundstellung mit dem Arbeitsplatz verbunden werden. Über diese Verbindung verfährt der Roboter gemeinsam mit dem Arbeitsplatz bei verschiedenen Höheneinstellungen und behält so eine feste Referenz gegenüber den anderen Arbeitsbereichen. Von seiner Position aus sind für den Roboter alle Arbeitsflächen des gesamten Aufbaus erreichbar. In diesem Arbeitsbereich sind nur Arbeitsprozesse für den Roboter geplant.

- Arbeitsbereich 3 definiert den rechten Schenkel des L-Aufbaus mit einer Arbeitsfläche der Aufnahme mit dazugehörigen Regalwagen zum Materialtransport. Der Regalwagen ist am Arbeitsbereich in der Grundstellung andockbar und gemeinsam mit dem gesamten Arbeitsplatz höhenverstellbar. Als zukünftige Erweiterung ist hier der Transport des Regalwagens über ein fahrerloses Transportsystem (FTS) möglich. In diesem Arbeitsbereich sind Tätigkeiten für MitarbeiterInnen und Roboter vorgesehen

Der gesamte Aufbau ist zur Ergonomieverbesserung in der Höhe verstellbar. Zur Versorgung des Arbeitsplatzes ist ein zentraler 16A Sicherungskasten mit Hauptschalter und ein Druckluftanschluss installiert. Die LED-Beleuchtung des Arbeitsplatzes wurde entsprechend der zusätzlichen Verwendung als Kameralicht dimensioniert. In Tab. 7.2 sind die eingesetzten Assistenzsysteme den Arbeitsbereichen zugewiesen und kurz definiert.

Tab. 7.2: Assistenzsysteme für den Anwendungsfall des Partners SPN

\begin{tabular}{l|l|l|l}
\hline Name & Art der Assistenz & Arbeitsbereich & Funktion \\
\hline Roboter & physisch & AB 1/2/3 & $\begin{array}{l}\text { Pick and Place in Zusammen- } \\
\text { arbeit mit dem/r MItarbeiterIn, } \\
\text { Auftragen der Dichtraupe }\end{array}$ \\
\hline Pick-by-light & kognitiv & AB 1/3 & $\begin{array}{l}\text { Kennzeichnung der benötigten } \\
\text { Materialkisten inkl. Mengenan- } \\
\text { gabe und Eingriffskontrolle }\end{array}$ \\
\hline Touchbildschirm & kognitiv & AB 1/3 & $\begin{array}{l}\text { Anzeige der Montageschritte } \\
\text { und Kommunikation mit der } \\
\text { zentralen Steuerung }\end{array}$ \\
\hline Intelligenter Schrauber & physisch & AB 1 3 & $\begin{array}{l}\text { Bauteilerkennung zur Roboter- } \\
\text { steuerung und Bildaufnahme } \\
\text { zur Qualitätssicherung }\end{array}$ \\
\hline Dosiersystem & physisch & AB 2 & $\begin{array}{l}\text { Montage der Schrauben mit } \\
\text { automatischer Drehmoment- } \\
\text { und Mengenüberwachung }\end{array}$ \\
\hline
\end{tabular}


Auf einem zentralen Rechnersystem läuft die Steuersoftware, in die die einzelnen Assistenzsysteme eingebunden und miteinander vernetzt sind. Hierüber finden zudem die Kommunikation und der Datenaustausch mit dem Firmennetzwerk statt. Alle notwendigen Montageinformationen und Meldungen werden den MitarbeiterInnen in einzelnen Arbeitsschritten dargestellt. Fertigungsprotokolle und Daten der Qualitätssicherung werden vom System zu jeder montierten Produktionseinheit automatisch abgespeichert.

Der aktuelle Arbeitsplatzaufbau ist in Abb. 7.11. zu sehen. Alle in Tab. 7.2 aufgeführten Assistenzsystemkomponenten (Slaves) sind an die zentrale Steuerungsplattform der paragon semvox GmbH als Master-System angebunden. Die Standardisierung des Montagefertigungsprozesses in der vorgegebenen Reihenfolge und deren kontinuierliche Überwachung durch Bestätigung der Bauteilentnahme bzw. der Weiterschaltung der standardisierten Arbeitsschritte gewährleistet eine erheblich gesteigerte Prozesssicherheit - auch bei mangelnder Verfügbarkeit von erfahrenem Montagepersonal.

Für den Anwendungsfall selbst wurde der Heizvorgang für die Getriebegehäuse auf 160 Grad Celsius in einen nicht zugänglichen Bereich verlegt, sodass der Werker keiner Gefahr durch zufälligen Körperkontakt mit der Heizplatte oder dem heißen Werkstück kommt. Das Handling des heißen Werkstückes wird nicht mehr durch den Menschen selbst, sondern durch den Roboter parallel zu den weiteren vorbereitenden Arbeiten des Werkers vollzogen. Das Einsetzen der Getriebewellen in die Bohrungen des erhitzten Gehäuses wiederum wird weiterhin durch MitarbeiterInnen ausgeführt, jedoch in einer ergonomisch günstigeren Position. Das heiße Bauteil wird anschließend vom Roboter zur Abkühlung in einen ungefährlichen Bereich abgelegt.

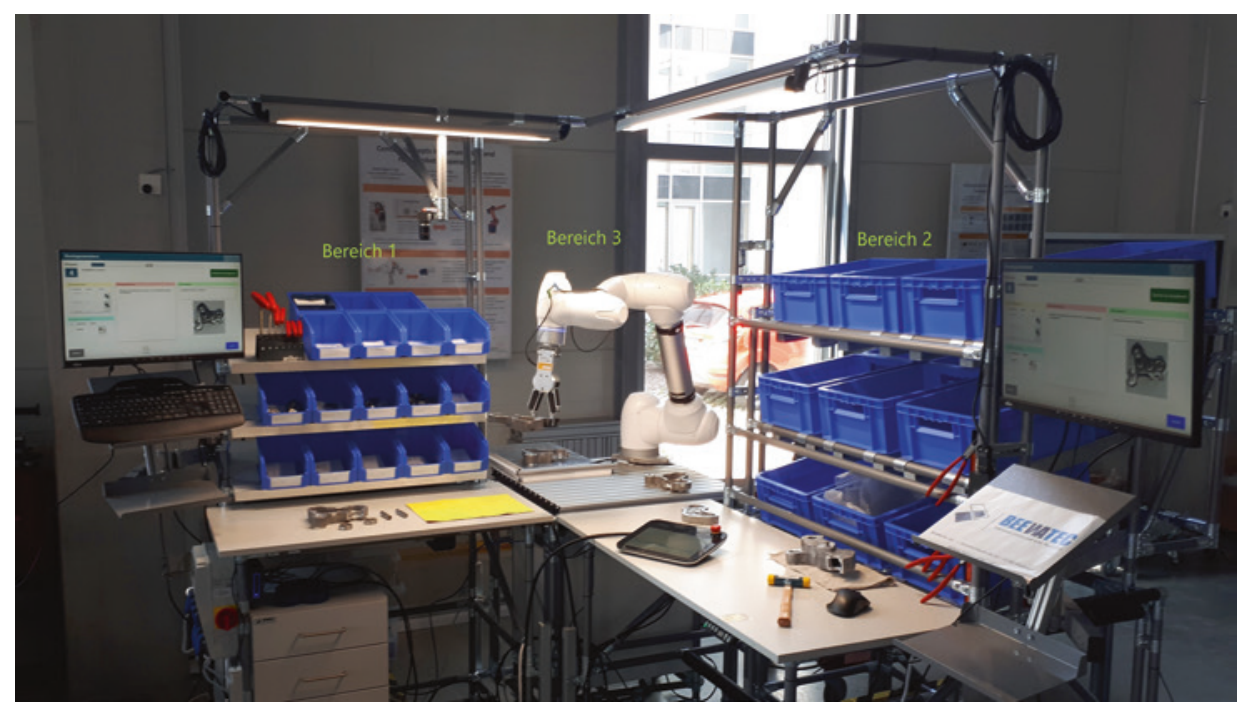

Abb. 7.11 Spezifizierter Montagearbeitsplatz 
Auch Arbeitsschritte mit hohen dauerhaften Anforderungen an Wieder- und Positionsgenauigkeit sowie feinmotorische Koordination werden durch physische Assistenzsysteme wesentlich vereinfacht und die Standardisierung und Prozesssicherheit so erheblich verbessert. Im Projekt SynDiQuAss wurde beispielhaft im Demonstrator der Arbeitsgang Dichtraupen-Auftragung auf Getriebegehäuse durch MRK-Roboter angesetzt.

\subsubsection{Umsetzung am Beispiel des zweiten Anwendungsfalls bei Ohnhäuser}

Der Industriepartner Ohnhäuser fertigt und montiert an dem betrachteten Arbeitsplatz Videoarme für Flugzeugsitze, die aus verschiedenen Unterbaugruppen bestehen. Die Unterbaugruppen Anbindung, Gelenk und Anbindung Bildschirm haben je ein verstellbares Rotationsgelenk. Zudem ist an der Unterbaugruppe Anbindung ein Entriegelungsknopf, der nach Betätigung das Ausklappen des Videoarms erlaubt. Um bei Turbulenzen während eines Fluges die nötige Sicherheit gewährleisten zu können, trotzdem aber die nötige Leichtgängigkeit der Gelenke zu ermöglichen, muss die benötigte Verstellkraft der Gelenke in einem bestimmten Toleranzbereich liegen. Diese Verstellkräfte wurden vor dem Projektstart manuell von dem/der MontagemitarbeiterIn gemessen. Die bisherige manuelle Ermittlung der Verstellkräfte war stark von der Verstellgeschwindigkeit während des Messvorgangs abhängig. Um eine höhere Reproduzierbarkeit zu gewährleisten, wurde ein robotergestützter Prüfprozess entwickelt. Eine besondere Herausforderung stellt hierbei die hohe Variantenvielfalt der Videoarme und die damit einhergehenden Variationen der Messvorgänge dar. Der Ablauf des assistierten Prüfprozesses ist wie folgt:

1. Bei dem Auftragseingang einer neuen Variante werden die Prozessrelevanten Parameter der einzelnen Unterbaugruppen ausgemessen und in einer Datenbank abgelegt.

2. In Bezug auf die Datenbank werden die vom Roboter anzufahrenden Punkte berechnet, in Robotertrajektorien zurücktransformiert und in eine.script Datei geschrieben

3. Die.script Datei kann daraufhin in das Roboterprogramm übernommen und abgespeichert werden.

4. Nach Abschluss der Montage wird das entsprechende Roboterprogramm der montierten Videoarmvariante aufgerufen und die Messung durchgeführt

5. Die gemessenen Kraftwerter werden automatisch als.csv Datei abgespeichert und Ausgewertet

6. Die Auswertung wird dem/der MonteurIn mit möglichen Anweisungen zur Nacharbeit angezeigt und in der Datenbank gespeichert.

In dem Anwendungsfall des Industriepartners Ohnhäuser ist der Arbeitsplatz mit fünf Modulen aufgebaut und mit unterschiedlichen Assistenzsystemen bestückt. Die ausgewählten Komponenten zur Assistenz des Industriepartners sind Touchscreens, ein 


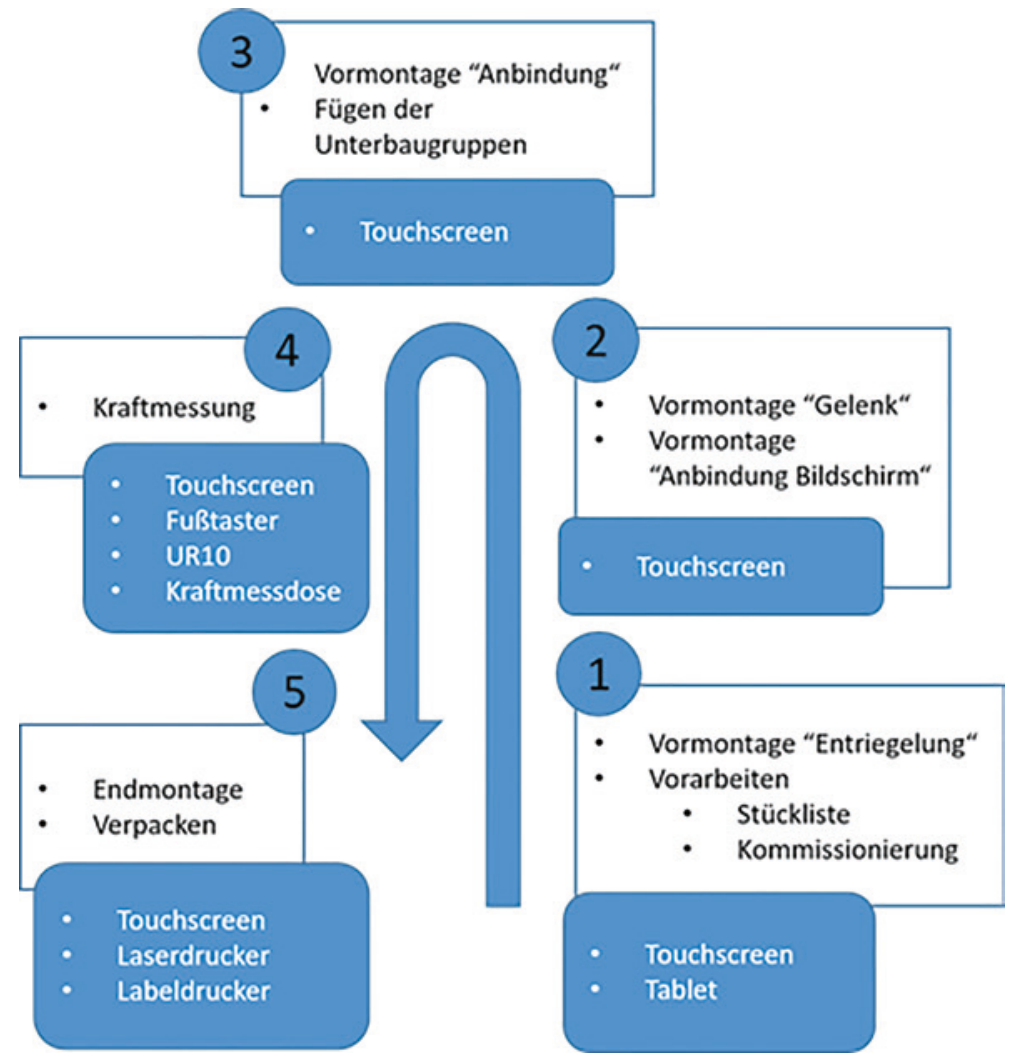

Abb. 7.12 Modularer Arbeitsplatz mit Assistenzsystem für Ohnhäuser

Tablet, ein Fußtaster, ein Laserdrucker und ein Etikettendrucker sowie für die Kraftmessung der UR10-Roboter und eine Kraftmessdose. Jedes der fünf Module ist mit Touchscreen-Monitoren ausgestattet, die den aktuellen Arbeitsstatus und Arbeitsanweisungen anzeigen. In den ersten drei Modulen werden die Unterbaugruppen vormontiert. Zudem werden die Unterbaugruppen an Modul drei zusammengefügt. Der UR10 mit der Kraftmessdose als Endeffektor befindet sich am vierten Modul. Der Fußtaster fungiert als Kommunikationskanal zwischen dem Monteur und dem paragon semvox-Assistenten. Am letzten Modul werden ein Laserdrucker und ein Etikettendrucker verwendet, um das Produkt zu kennzeichnen und die Qualitätsdokumentation auszudrucken, siehe Abb. 7.12.

\subsubsection{Softwaresystem für Montageassistenz}

Die während des Projektes entwickelte Software besteht aus einem Assistenten für Montageaufgaben und einen Editor zur Erzeugung und Anpassung von beliebigen 
Instanzen des intelligenten Assistenten. Beide Applikationen basieren auf einem gemeinsamen Grundkonzept, der zum einen den State of the Art der Interaktionswissenschaft berücksichtigt und zum anderen die besonderen Anwendungsanforderungen von SynDiQuAss und der Anwendungsszenarien bedient. Entsprechend der Anforderungsanalyse konkretisierte sich als Ziel des Projektes eine Software, die sich möglichst einfach bedienen lässt, um Umschulungen von MitarbeiterInnen zu vermeiden, und die Erstellung von neuen Instanzen von Assistenten für unterschiedlichen Versionen von Produkten ermöglicht, damit die Wirtschaftlichkeit des Einsatzes auch für die Produktion von kleineren Produktstückzahlen gewährleistet ist.

\subsubsection{Gesamtkonzept}

Ein Datenmodell, das den Prozessworkflow bei der Montage abbildet, dient als gemeinsame Grundlage für den Assistenten und für den Editor. Demnach besteht der Montageassistenzprozess aus mehreren Prozessschritten, die den Aktivitäten des Prozessmodells entsprechen (vgl. Abschn. 7.3.3.1). Jeder Prozessschritt sieht folgende Funktionalität vor:

- dem Arbeiter werden Prozessinstruktionen in der GUI gezeigt (Text, Bild).

- bei jedem Prozessschritt kann dann der Arbeiter über mehrere zu seiner Wahl stehenden Modalitäten (Sprache, Touchscreen, Fußpedal) zu den jeweiligen Schritten vor und zurücknavigieren ("zurück/weiter").

- bei sensiblen Prozessschritten (z. B. wenn eine Überprüfung der Teile notwendig ist, weil der Arbeitsschritt unumkehrbar ist) wird eine Bestätigung des Arbeitsschrittes vom System verlangt. Nur dann kann der Arbeiter weiter navigieren.

- bei jedem Schritt kann der Arbeiter nach Hilfe verlangen, die in Form z. B., von Video-aufzeichnungen angeboten wird (siehe GUI).

- bei jedem Schritt kann der Arbeiter Kommentare sprachlich eingeben, die in den Montageanweisungsbereich (Siehe GUI) übernommen werden. Ein übergeordneter Nutzer soll diese Kommentare editieren können (z. B., Löschen, Erweitern, Anpassen).

- es sollen mehrere Zugriffsrechte mit dedizierten Funktionen für die Applikation vorgesehen werden (z. B., Editor, Werker).

Das Datenmodell sieht also die Definition von abstrakten, semantisch realisierten Datenstrukturen (Konzepten in einer Ontologie) für folgende Elemente vor (Abb. 7.13):

- Prozessschritt

- DisplayObject

- Benötigte Teile

- Montageanweisung

- Hilfeanweisung

- Unterprozessschritte 


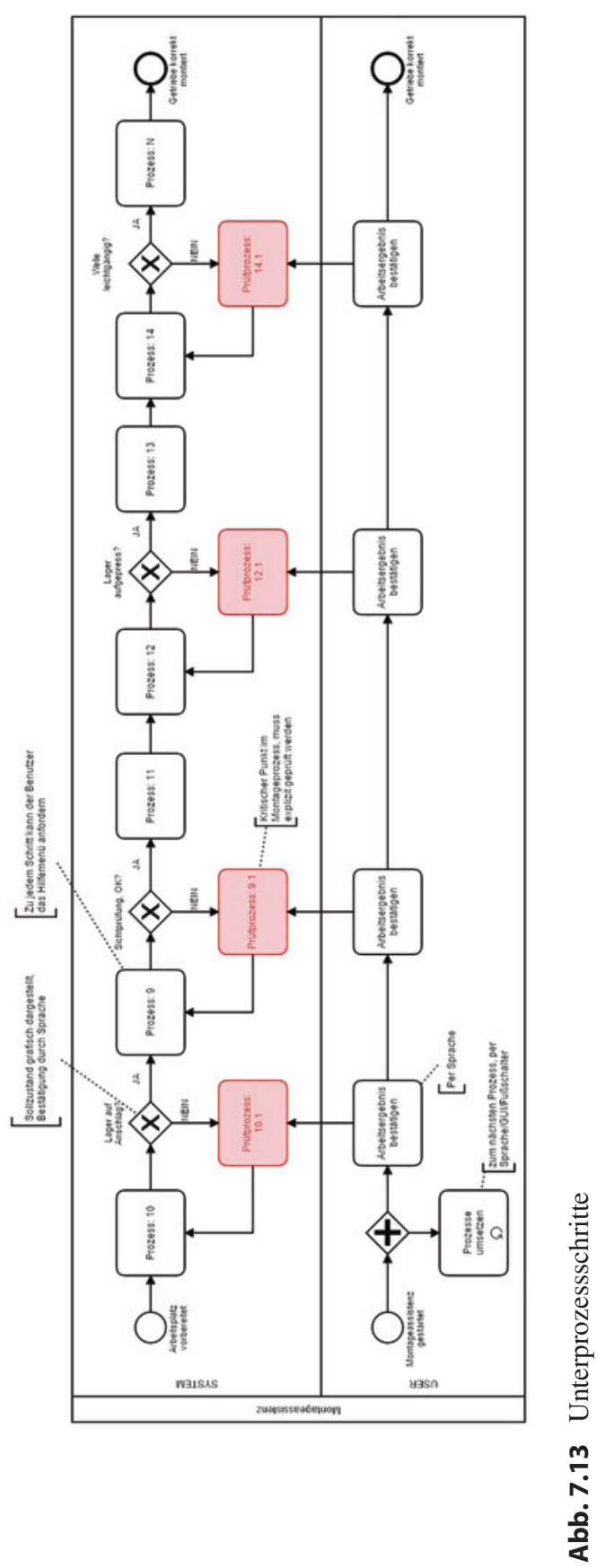


Instanzen des semantisch abstrahierten Datenmodels werden im Excel Format persistiert und stellen nun die Referenzablage für Speicherung der Daten im Editiermodus und für Wiedergabe der Assistenz beim Assistenzmodus.

Für die Interaktion wurde ein multimodaler und multimedialer Ansatz vorgenommen.

Multimodalität gewährleistet die freie Wahl der zu nutzenden Modalität (z. B., Gestensteuerung, sprach- und keyboardbasierte Interaktion, Touchscreeneingabe) für den Mitarbeiter und somit einen einfachen Zugang von unerfahrenen Personal zum Tool.

Multimedialität sorgt wiederum für eine vollständige Nutzung des zur verfügungsstehenden Informationsmaterial (z. B. Abbildungen und Bezeichnungen der zu montierenden Teile, Videoaufnahmen von beispielhaften Montageschritten, Beschreibungen der Montageschritte in Text und Sprachformat).

\subsubsection{Spezifikation der Funktionalitäten des Softwaresystems}

Da den MitarbeiterInnen multimodale Interaktionsmöglichkeiten zur Verfügung gestellt werden sollen, sieht der angezielte Testarbeitsplatz mehrere Interaktionskomponenten vor:

- Touchbildschirm zur Visualisierung der Prozessschritte und Interaktion über dedizierte grafische Benutzerschnittstelle GUI

- Mikrofone und Lautsprecher für die Sprachinteraktion

- Instrumentierten (pick by light) Schüttboxen zur Erkennung der einzusetzenden Bestandteile.

Die GUI zur visuellen Darstellung der Interaktion ist in funktionsabhängige Bereiche gegliedert, um die kognitive Last bei der Interaktion zu minimieren. Vertikal geordnet sind die Hauptbereiche, die jeweils den Prozessverlauf und Zustand, die Prozessschrittanleitung und die Prozessnavigation abbilden. Auf Prozessverlauf und Zustandsebene sind dann Informationen zu Prozessschritt, Prozessverlauf und Bestätigungsfunktionalität horizontal eingeordnet. Die Prozessschrittanleitungsebene sieht dann horizontale Bereiche für die Angaben von benötigten Bauteilen und Werkzeugen, Montageanweisung und Sollzustand vor. Die Funktionalitäten zur Navigation in den jeweiligen Schritten (weiter und zurück) und die Hilfefunktionalität mit Zugriff auf multimediale Inhalte sind $\mathrm{m}$ Navigationsbereich abhängig von den vorhandenen Medien (Bilder, Videos, Zeichnungen usw.) horizontal eingeordnet (Abb. 7.14).

\subsubsection{Spezifikation der Editorfunktionalität}

Nach der Identifikation der zur Editierung benötigten Daten und der an dem Editierprozess beteiligten MitarbeiterInnen wurde ein Konzept für den Editor implementiert. Dieser ermöglicht den Aufbau der jeweiligen Assistenzinstanzen durch die Eingabe der 


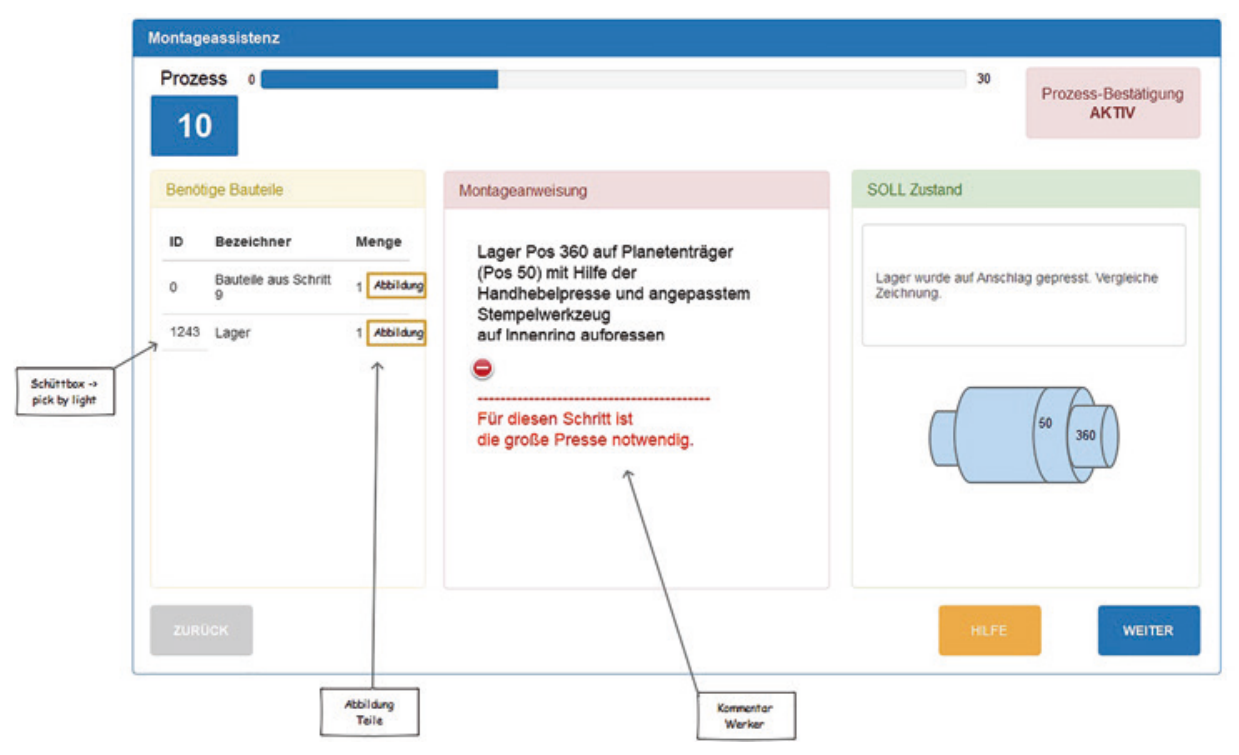

Abb. 7.14 Beispiel für den Montageassistenten

produktfamilienspezifischen Instruktionen von MitarbeiterInnen mit unterschiedlichen Zugriffsrechten. Hierbei stellte sich auch heraus, dass viele Daten wohl am Anfang des Prozesses schon vorhanden sind und zur Nutzung im Editiermodus automatisch eingetragen oder bereitgestellt werden. Daraus resultiert ein bimodaler Ansatz für den Assistenzsystemeditor:

1. Datengetriebene Eingabe: Der Editor erzeugt automatisch aus schon vorhandenen Dokumentationsmaterialien fertige, weitereditierbare Prozessassistenzinstanzen. Dies ist in Form von dedizierten Plug-ins realisiert (Semantic Lifting).

2. MitarberInnengetriebene Eingabe: Der Editor soll allen MitarbeiterInnen, die an der Entstehung eines Arbeitsprozesses beteiligt sind, dedizierte Editiermöglichkeiten anbieten. Hierbei wird in Interaktion mit dem Assistenzsystem selbst der Prozess erzeugt.

Auf der Basis der Anforderungen wurde ein Editierprozess zur semantischen Repräsentation spezifiziert, der sich aus dem Editierprozess (Sequenzierung, Identifikation der Teile, Identifikation der Werkzeuge, Erklärung der Schritte, Hinzufügen von multimedialem Material) selbst sowie dere Nacheditierung (Korrektur der zuvor eingegebenen Informationen, Anreicherung von zusätzlichen Informationen, Umeditierung zum Zweck der Herstellung von zusätzlichen Varianten des Editierprozesses für verwandte Produkte) zusammensetzt. 


\subsubsection{Implementierung}

Der Montageprozessassistent und -editor werden auf der Basis der paragon semvox ODP (Ontologie-basierte Dialog Plattform) Interaktionsmanagement Plattform und der Plattform für verteilte Intelligenz semvox.AI umgesetzt. Semvox.AI wiederum stellt die aktuellste Generation der von paragon semvox angebotener Interaktionsplattform dar. ODP S. 3 bietet hierbei einen vollständigen Technologiestack für dialogisches Interaktionsmanagement und kombiniert mehrere Methoden der multimodalen Verarbeitung. Die semvox.AI unterstützt die kognitive Architektur eines intelligenten Assistenten als zusätzlicher modularer Layer zu ODP S. 3 zur Ableitung und Verwaltung von zusätzlichem Wissen und Kontextinformationen aus verteilten intelligenten Diensten.

Interaktionsmanagement Plattform und der Plattform für verteilte Intelligenz semvox. AI umgesetzt und besteht aus folgenden Komponenten:

- Interaktionsmanager

- Spracherkennung und Sprachsynthese

- Gestenerkennungskomponente

- GUI

- Schnittstellen zu Externedatenbanken

- Externe Sensorik (Mikrofon, Kamera/Eyetracking, Leapmotion, Pick by Light System)

Der Prozess und die Prozessschritte werden in diesem Tool in Form von semantischen Objekten als Tasks realisiert. In dem ODP Framework werden dann Datenanreicherung, multimodale Fusion, Interaktionsmanagement, Aktionsplanung sowie multimodale Fission (Generierung) ausgeführt. Die GUI wird auf der Basis der javafx Bibliotheken als eigenständige Komponente realisiert und ist sowohl für die Aufnahme und Weiterleitung von Touch- und Text Input-Eingaben zur ODP-Fusion, als auch für die Umsetzung von visuellen Angaben, auskommend von der ODP-Generierung verantwortlich. Die Gestenerkennungskomponente ist auf der Basis von erweiterten mitgelieferten java-Interfaces zum Leap-Motion Sensor realisiert. Die Kommunikation zwischen ODP und externen Komponenten findet auf der Basis von Java statt.

Spracherkennung und Sprachsynthese werden auf Basis der Nuance Technologie, jeweils über das Vocon Hybrid embedded ASR (Spracherkennung) und embedded Vocaliser TTS (Sprachsynthese) umgesetzt. Bei der Spracherkennung wird auch auf die spezielle „embedded“, open Domain Diktierfunktion zurückgegriffen, womit die Eingabe von Kommentaren oder Prozessschritte-Beschreibungen umgesetzt wird.

Für die Prozesseditierung werden in dem ursprünglichen Assistenten Excel-Dateien (für jede Datei ein Montageprozess) verwendet, die einfach strukturiert und übersichtlich als eine gute allgemein editierbare Grundlage dienen. Die jeweilige Excel-Datei wird dann automatisch in eine entsprechende ODP-Montageprozessinstanz beim Start der Applikation umgewandelt und beim Editierprozess dynamisch angepasst. Dies wird weiter als Basisdatenablage genutzt (Abb. 7.15). 


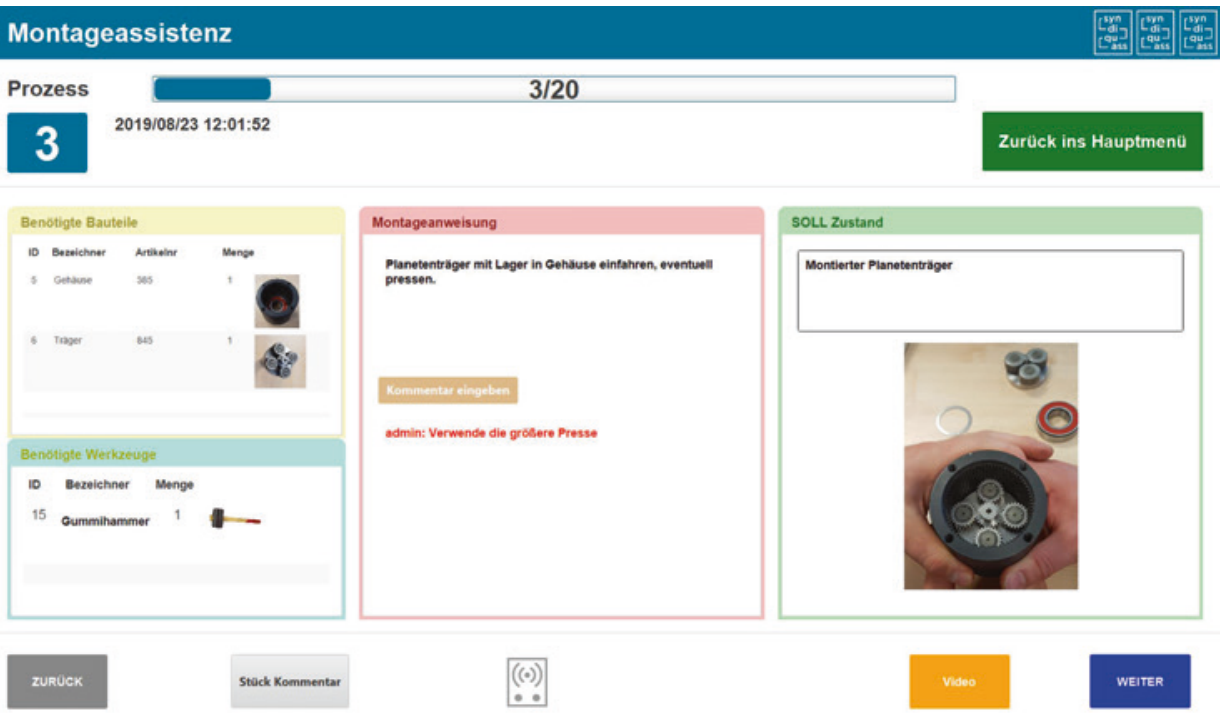

Abb. 7.15. Beispiel für den Montageassistenten

Eckdaten zum System:

- Sprache: Deutsch

- Betriebssystem: Windows

- Falls Linux: Also ab Version 1.0.29

- Java 32 Bit ab Version 1.8

- Hardware: Laptop/Raspberry Pi

- Spracherkennung: Nuance VoCon 4.11.13

- Sprachsynthese: Nuance Vocalizer 3.0.

\subsection{Lessons Learned}

Aus der Zusammenarbeit innerhalb des Projekts lassen sich wertvolle Erkenntnisse hinsichtlich der Organisation, Zusammenarbeit, Ergebnisse und deren Verwertbarkeit ableiten.

Lesson 1: Das durch das Commitment definierte partizipatorische Modell ist ein wesentlicher Erfolgsfaktor bei der Umsetzung technologischer Transformationsprozesse. Dies gilt insbesondere für bestehende und langjährig gewachsenen Strukturen in Unternehmen

Technologische Transformationsprozesse werden oftmals durch disruptive Innovationen ausgelöst oder begleitet. Die Komplexität der Assistenzsysteme ist nur schrittweise und 
in begrenzt zumutbaren Umfang zu steigern, damit sich die Menschen nicht zu schnell überfordert fühlen und die Vorteile der Assistenz nicht mehr wahrnehmen können. Zu exotisch anmutende Features der High-Tech-Industrie müssen gut auf die gewählte Zielgruppe zugeschnitten sein, um nicht abgelehnt zu werden. In jedem Fall muss die Prozesssicherheit und gewünschte Funktionalität der neuen Technologien gewährleistet und zumindest jederzeit kurzfristige Unterstützung bei Problemen verfügbar sein. Genügend Ressourcen an Zeit und Manpower für die Einführung und Schulung der Anwender ist grundsätzlich notwendig für den produktiven Einsatz.

Ohne die enge Kommunikation mit allen Betroffenen auf allen Ebenen der Unternehmenshierarchie werden die unterschiedlichen Ideen und Lösungsansätze zur Zielerreichung nicht aufgenommen und die einzuführenden Prozessänderungen nicht in der Praxis umgesetzt. Authentische und offene Auseinandersetzung mit den Anforderungen und Bedenken der Anwender ist dabei zu beachten. Wichtig ist dabei auch die Zusammenarbeit und Einbeziehung des Betriebsrates als Arbeitnehmervertretung von Beginn an.

Ein ständiger Austausch mit Marktteilnehmern in ähnlichen Aufgabenstellungen über die Branchengrenzen und Region hinaus bringt neue Gedankenansätze und Einblicke in Lösungsstrategien anderer Organisationen, die weiterhelfen können. Auch die musterhafte Darstellung und eigene Probiererfahrungen in speziell eingerichteten Instituten kann erheblich zum Abbau von Vorbehalten und zur Begeisterung beim direkten Erleben der Funktionen beitragen.

Beispiele hierfür konnten im Projekt SynDiQuAss folgende Einrichtungen bieten:

Future Work Lab am Fraunhofer IAO in Stuttgart

Lernfabrik für vernetzte Produktion LVP am Fraunhofer IGCV in Augsburg

Technologietransferzentrum (TTZ) der Hochschule Augsburg am TCW in Nördlingen

\section{Lesson 2: Die Zusammensetzung des Projektkonsortiums sollte den Querschnitt aus Industrie-, Technologie- und Forschungspartnern repräsentieren, um die spezi- fischen Herausforderungen der digitalen Transformation abzubilden}

Für die erfolgreiche Umsetzung der digitalen Transformation muss domänenspezifisches Expertenwissen mit domänenübergreifender Methodenkompetenz kombiniert werden. Produkt- und prozessspezifisches Wissen liegt an zahlreichen Stellen, meist jedoch nicht in strukturierter und digitalisierter Form in den Industrieunternehmen vor. Dessen Nutzung und Nutzbarmachung stellt oftmals den Einstieg in die Digitalisierung kleiner und mittelständischer Unternehmen dar. Als Ansatzpunkt zur weiteren Verbreitung sind Handlungsempfehlungen gerade für die Informations- und Datenaufbereitung und-speicherung sowie die IT-Struktur und Prozessabläufe von großer Bedeutung.

Dies gilt ganz besonders für die Entwicklung digital vernetzter Assistenzsysteme. Hierfür sind Fachkenntnisse aus dem jeweiligen Produktionsprozess zur Gestaltung der Unterstützung, betriebswirtschaftliche Kenntnisse zur Bewertung der Investitionsentscheidung, juristische Kenntnisse zur datenschutz- und arbeitsrechtlichen Bewertung sowie IT-Kenntnisse zur Unternehmensanbindung von Nöten. Gerade bei dem 
Zusammenspiel zwischen Produktion und IT konnten im Projekt SynDiQuAss wertvolle Erfahrungen gesammelt werden, die mit dieser Veröffentlichung weitergegeben werden sollen.

Es ist hierbei unerlässlich, dass bei der Neuentwicklung eines Assistenzsystems eine Begehung des Arbeitsplatzes durch Softwareentwickler erfolgt. Gerade in der Montage muss bei den Entwicklern ein Eindruck vorhanden sein wie sich das jeweilige Produkt anfühlt, welche Werkzeuge verwendet werden, wie die Arbeitsatmosphäre vor Ort ist.

Lesson 3: Der Entwicklungsprozess in interdisziplinären Teams muss moderiert werden, um die Zielerreichung für das Gesamtprojekt sicherzustellen. Dabei sind Entwicklungstools z. B. aus den Bereichen der agilen Softwareentwicklung hilfreich Im Forschungsprojekt SynDiQuAss übernahm das Fraunhofer IGCV die Rolle des Vermittlers, um Aufwand und Nutzen während der Softwareentwicklung des Montageplaneditors zu bewerten. Ziel des Vermittlers war es diejenigen Softwareanforderungen zu identifizieren, die mit geringem Aufwand einen hohen Nutzen haben und diejenigen Softwareanforderungen zurückzustellen, die trotz hohem Aufwand nur einen geringen Nutzen abwerfen.

Zur Verwaltung der Softwareanforderungen wurde bei der Assistenzsystementwicklung im Projekt SynDiQuAss ein Tool aufgesetzt, in das regelmäßig neue Anforderungen eingetragen worden. Dieses Tool wurde zudem genutzt, um Priorität und Status zu tracken. In wöchentlichen Telefonkonferenzen wurden mit dem Softwarepartner Änderungen besprochen. Das iterative Entwicklungsvorgehen durch das im zweiwöchentlichen Rhythmus neue Releases der Software erschienen sind hat sich als bewährt herausgestallt. Hierdurch konnten Änderungen an der Usability zeitnah getestet werden und die Integration von Hardwarekomponenten Schritt für Schritt vorgenommen werden. Durch die Verkürzung des Release-Zyklus auf zwei Wochen konnte im Projekt ein enormer Anstieg der Entwicklungsgeschwindigkeit im Vergleich zu frühen Projektphasen beobachtet werden.

Lesson 4: Als zentrales Projektergebnis entstehen Technologiedemonstratoren. Diese dienen sowohl der projektinternen Verwertung, d.h. als Grundlage für Assistenzsysteme in der Montage der Projektpartner, als auch der externen Verbreitung und Verfügbarkeit, z. B. in Form eines Weiterbildungsangebotes für interessierte Unternehmen

Das Projekt SynDiQuAss ist mit der Zielsetzung gestartet, anwendungsnahe Technologiedemonstratoren für die digital vernetzte Assistenz an Montagearbeitsplätzen zu entwickeln und im Praxisbetrieb zu evaluieren. Während der Laufzeit wurden deshalb u. a. ein Demonstrator für die Technologie- und Akzeptanzuntersuchung von Assistenzsystemen (Fraunhofer IGCV) als auch ein Systemarbeitsplatz mit kollaborierendem Roboter (Hochschule Augsburg) aufgebaut, mit dem assistierte Qualitätsprüfungsaufgaben und die kollaborierende Montage getestet werden. Neben der Evaluation und Konzeptvalidierung ist die spätere Verwertung der Demonstratoren ein wichtiges 
Projektziel. So dienen diese einerseits den Forschungsinstituten als Demonstrationsobjekte, die für weitere FuE-Vorhaben, aber auch für die Entwicklung eines maßgeschneiderten Weiterbildungsprogramms verwendet werden. Auf diese Weise wird sichergestellt, dass sich Projektergebnisse über den Kreis des Konsortiums hinaus verbreiten. Andererseits stellen die evaluierten Demonstratoren die Grundlage dafür dar, in für den Produktiveinsatz weiterentwickelter Form in die Montagebereiche der beteiligten Industriepartner integriert $\mathrm{zu}$ werden und damit zukünftig für praktisch messbare Produktivitätssteigerungen und Qualitätsverbesserungen zu sorgen.

Lesson 5: Der technologische Reifegrad lässt den Einsatz der im Projekt SynDiQuAss entwickelten Technologiedemonstratoren innerhalb von Forschungsund Testuntersuchungen zu. Um die Systeme für den Produktivbetrieb nutzen zu können, sind Weiterentwicklungen insbesondere im Bereich der Arbeitssicherheit erforderlich. Die Projektergebnisse der Konzeptions- und Testphase liefert dazu bereits hilfreiche Erkenntnisse

Der Reifegrad der Demonstratoren erlaubt den Einsatz unter Forschungs- und Testbedingungen. Für den Produktivbetrieb sind jedoch weitere Entwicklungsstufen $\mathrm{zu}$ durchlaufen. Insbesondere arbeitsschutzrechtliche Bestimmungen (Maschinenrichtlinie, CE-Kennzeichnung) müssen mit geeigneten Verfahren wie einer ausführlichen Gefahren- und Risikoanalyse abgesichert werden. Dies gilt insbesondere für kollaborierende Assistenzsysteme, der WerkerInnen physisch unterstützen. Im Rahmen des Demonstratoraufbaus werden deshalb bereits Auslegungskriterien wie Bahngeschwindigkeiten und die sichere Interaktion zwischen Bediener und Assistenzsystem untersucht. Diese Projektergebnisse stehen den Konsortialpartnern für Weiterentwicklungen zur Verfügung. Dennoch besteht im Bereich der sicheren Applikationsentwicklung kollaborierender Systeme noch Forschungsbedarf, um Anwendern die Planung, Auslegung und praktische Nutzung solcher Systeme zu vereinfachen.

\section{Literatur}

1. Adler, S.; Böhme, T.; Möser, S.: Assistenzsysteme in der Anlagen-Inbetriebnahme. wt- online 106 (2016) 3, S. 117-118.

2. Apt E, Bovenschulte M, Priesack K, Weiß Chr, Hartmann A (2018) Einsatz von digitalen Assistenzsystemen im Betrieb, iit - Institut für Innovation und Technik, Berlin

3. Bannat A (2014) Ein Assistenzsystem zur digitalen Werker-Unterstützung in der industriellen Produktion. Dissertation, Technische Universität München

4. Bauernhansl T Hompel M ten, Vogel-Heuser B (2014) Industrie 4.0 in Produktion, Automatisierung und Logistik - Anwendung, Technologien, Migration. Springer Vieweg, Wiesbaden

5. Bender M (2016) Leichtbauroboter in der manuellen Montage - einfach einfach anfangen: Erste Erfahrungen von Anwenderunternehmen. Stuttgart. Zugegriffen: 23.07.2019 
6. Bischoff J, Taphorn C, Wolter D, Braun N, Fellbaum M, Goloverov A, Scheffler D: Erschließen der Potenziale der Anwendung von Industrie 4.0 im Mittelstand, agiplan, Mülheim an der Ruhr

7. Blutner D, Cramer S, Krause S, Mönks T, Nagel L, Reinholz A, Witthaut M (2009) Assistenzsysteme für die Entscheidungsunterstützung. In: Buchholz, P. et al. (Hrsg.): Grosse Netze der Logistik, Springer, Berlin, S. 241-270.

8. Buchholz B, Ferdinand J.-P, Gieschen J.-H., Seidel U (2017) Digitalisierung industrieller Wertschöpfung - Transformationsansätze für KMU, VDI/VDE Innovation + Technik GmbH, Berlin

9. Bullinger H.-J. (1995) Arbeitsplatzgestaltung - Personalorientierte Gestaltung marktgerechter Arbeitssysteme, Springer Vieweg, Wiesbaden

10. Davis F (1985) A technology acceptance model for empirically testing new end-user information systems - theory an results, $\mathrm{PhD}$ thesis, Massachusetts Inst. Of Technology

11. Deuse J, Busch F, Weisner K, Steffen M (2015): Differentielle Arbeitsgestaltung durch hybride Automatisierung. In: Schlick Chr (Hrsg.) Arbeit in der digitalisierten Welt, Frankfurt/ New York, S. 235-246

12. DIN ISO/TS 15066:2016, Februar 2016 (2016) Roboter und Robotikgeräte - Kollaborierende Roboter

13. Drath R, Fay A, Barth M (2011) Interoperabilität von Engineering-Werkzeugen. In: at - Automatisierungstechnik 59, S. 451-460.

14. Eigner M, Stelzer R (2013) Product Lifecycle Management; Ein Leitfaden für Product Development und Life Cycle Management. Springer Verlag. Heidelberg, London, New York

15. Feldmann K, Slama S (2001) Effizienzsteigerung in der Montage durch marktorientierte Strukturen und erweiterte Mitarbeiterkompetenz. In: wt Werkstatt online 91, 8. Springer-VDI-Verlag, S. 483-488

16. Funk, M.; Schmidt, A.: Cognitive Assistance in the Workplace. IEEE Pervasive Computing 14 (2015) 3, S. 53-55.

17. Hinrichsen, Sven, Riediger, Daniel \& Unrau, Alexander 2016. Assistance Systems in Manual Assembly.

18. Kargl H (2017) Kompendium zu Enterprise Architect von SparxSystems; Foundational, Sparx Systems Software GmbH, Wien

19. Kerber F, Haendel G (2016) A UML-based approach to manage product variability in automated production lines 2016 IEEE 21st International Conference on Emerging Technologies and Factory Automation (ETFA). IEEE, S. 1-5.

20. Kiem R (2016) Qualität 4.0 - QM, MES und CAQ in digitalen Geschäftsprozessen der Industrie 4.0. Carl Hanser Verlag, München

21. Klocke F, (2017) Vernetzte, adaptive Produktion, In: Internet of Production für agile Unternehmen, AWK Aachener Werkzeugmaschinen-Kolloquium 2017, Apprimus Verlag, S. 263286.

22. Klocke F, Kamps S, Mattfeld P, Shirobokov A, Stauder J, Trauth D, Basset E, Jurke B, Bönsch C, Gärtner R, Holsten S, Jamal R, Kerzel U, Stautner M (2017): Assistenzsysteme in der Produktionstechnik. In: Jamal R, Heinze R (Hrsg.) Virtuelle Instrumente in der Praxis VIP 2017. VDE Verlag, Berlin, S. 265-287

23. Lüder A, Schmidt N (2017) AutomationML in a Nutshell. In: Vogel-Heuser B, Bauernhansl T, Hompel M ten (Hrsg) Handbuch Industrie 4.0. Bd. 2, S. 213-258.

24. Ludwig B (2015) Planbasierte Mensch-Maschine-Interaktion in multimodalen Assistenzsystemen, Springer Verlag, Berlin, Heidelberg

25. McDermott R. E., Mikulak R. J., Beauregard M. R. (2019) The basics of FMEA, $2^{\text {nd }}$ edition, CRC Press, Productivity Press: S. 9 ff. 
26. Müller R, Vette M, Mailahn O, Ginschel A, Ball J (2014) Innovative Produktionsassistenz für die Montage. wt-online 104 - 9, S. 552-560.

27. Obermaier R (2016) R.: Industrie 4.0 als unternehmerische Gestaltungsaufgabe. Betriebswirtschaftliche, technische und rechtliche Herausforderungen. Springer Verlag. Berlin, Heidelberg

28. Object Management Group (2017) OMG Unified Modeling Language (OMG UML), Version 2.5.1

29. Prenting TO, Battaglin RM (1964) The precedence diagram: A tool for analysis in assembly line balanc-ing. In Journal of Industrial Engineering, S. 208-213.

30. Reinhart G, Zäh M. F, Wiesbeck M, Egbers J(2009) Digitale Assistenzsysteme zur alterungsgerechten Integration von Werkern in die variantenreiche Montage. ATZproduktion 2, 3-4, S. $18-21$.

31. Rößler A (2016) Management-Handbuch, b-wise, Karlsruhe

32. Rumpe B (2011) Modellierung mit UML; Sprache, Konzepte Und Methodik. Springer Verlag, Berlin, Heidelberg

33. Senderek R, Geisler K (2015) Assistenzsysteme zur Lernunterstützung in der Industrie 4.0. In: Rathmayer S, Pongratz H (Hrsg.) Proceedings of DeLFI Workshops 2015 co-located with 13th e-Learning Conference of the German Computer Society (DeLFI 2015), München, S. 36-46

34. Sochor R, Riegel A, Merhar L, Rusch T, Merkel L, Kerber F, Braunreuther S, Reinhart G (2019) Kognitive und physische Assistenz in der Montage, wt-online 3-2019, Seite 122-127.

35. Sommerhoff B (2015) Eine neue Ära der Qualitätssicherung. FAZ Frankfurter Allgemeine Zeitung. Online-Archiv. Zugegriffen: 23.07.2019

36. Steil J. J., Maier G. W. (2018) Kollaborative Roboter. Universale Werkzeuge in der digitalisierten und vernetzten Arbeitswelt. In: Maier G.W., Engels G, Steffen E (Hrsg.): Handbuch Gestaltung digitaler und vernetzter Arbeitswelten, Springer, Berlin

37. Ullrich A, Vladova G, Gronau N, Jungbauer N (2016) Akzeptanzanalyse in der Industrie 4.0-Fabrik. Ein methodischer Ansatz zur Gestaltung des organisatorischen Wandels. In: Obermaier, R. (Hrsg.): Industrie 4.0 als unternehmerische Gestaltungsaufgabe, Wiesbaden: Springer Gabler, 2016, S. 291-307

38. Ullrich C, Aust M, Blach, R, Dietrich M, Igel C, Kreggenfeld N, Kahl D, Prinz C, Schwantzer S (2015): Assistenz- und Wissensdienste für den Shopfloor. In: Rathmayer S, Pongratz $\mathrm{H}$ (Hrsg.) Proceedings der Pre-Conference Workshops der 13. E-Learning Fachtagung Informatik, München, S. 47-55

39. Venkatesh V, Morris M. G., Davis G. B., Davis F. D. (2003): User Acceptance of Information Technology: Toward a Unified View. MIS Quarterly, 37 (3), 425-478.

40. Weigert G, Henlich T, Klemmt A (2008) Methoden zur Modellierung und Optimierung von Montageprozessen. In: Rabe M (Hrsg) Advances in simulation for production and logistics applications. Tagungsband zur 13. Fachtagung, Berlin, S. 479-488.

41. Westkämper E, Löffler C (2016) Strategien der Produktion - Technologien, Konzepte und Wege in die Praxis, Springer Viehweg, Berlin, Heidelberg

42. Westphal A (2011) Ethikbasierte Unternehmensführung und Commitment der Mitarbeiter, Gabler Verlag, Springer Fachmedien, Wiesbaden

43. Wischmann S, Hartmann E.A (2018) Zukunft der Arbeit. Springer-Vieweg, Berlin 
Open Access Dieses Kapitel wird unter der Creative Commons Namensnennung 4.0 International Lizenz (http://creativecommons.org/licenses/by/4.0/deed.de) veröffentlicht, welche die Nutzung, Vervielfältigung, Bearbeitung, Verbreitung und Wiedergabe in jeglichem Medium und Format erlaubt, sofern Sie den/die ursprünglichen Autor(en) und die Quelle ordnungsgemäß nennen, einen Link zur Creative Commons Lizenz beifügen und angeben, ob Änderungen vorgenommen wurden.

Die in diesem Kapitel enthaltenen Bilder und sonstiges Drittmaterial unterliegen ebenfalls der genannten Creative Commons Lizenz, sofern sich aus der Abbildungslegende nichts anderes ergibt. Sofern das betreffende Material nicht unter der genannten Creative Commons Lizenz steht und die betreffende Handlung nicht nach gesetzlichen Vorschriften erlaubt ist, ist für die oben aufgeführten Weiterverwendungen des Materials die Einwilligung des jeweiligen Rechteinhabers einzuholen.

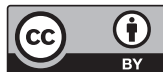

